\title{
Hybrid Algorithm for Rough Multi-level Multi-objective Decision Making Problems
}

\author{
Shereen Fathy El-Feky ${ }^{1}$, Tarek H.M. Abou-El-Enien ${ }^{2 *}$ \\ ${ }^{1}$ Teaching Assistant at Faculty of Computer Science, Department of Computer Science, Modern Science and Arts University, Giza \\ 12613, Egypt \\ ${ }^{2}$ Department of Operations Research \& Decision Support, Faculty of Computers \& Information, Cairo University, Giza 12613, \\ Egypt
}

Corresponding Author Email: t.hanafy@fci-cu.edu.eg

https://doi.org/10.18280/isi.240101

Received: 28 October 2018

Accepted: 12 January 2019

\section{Keywords:}

compromise programming, rough programming, TOPSIS method, global criterion method, multi-objective programming, multi-level programming

\begin{abstract}
The purpose of this paper is to generate compromise solutions for the multi-level multiobjective decision making (MLMODM) problems with rough parameters in the objective functions (RMLMODM) based on TOPSIS method and "Lower \& Upper" approximations method. We introduce a computational hybrid algorithm for solving RMLMODM problems. Also, we solved illustrative numerical example and compared the solution of the proposed algorithm with the solution of Global Criterion (GC) method. The engineers and the scientists can apply the introduced hybrid algorithm to various practical RMLMODM problems to obtain numerical solutions.
\end{abstract}

\section{INTRODUCTION}

Rough set theory is an important mathematical tool for dealing with the description of vague objects. Rough set methodology has been introduced by Pawlak (1991), [1].

Linear optimization problem which is considered where some or all of its coefficients in the objective function and/or constraints are rough intervals is introduced by Hamzehee et al. [2].

Various hybrid algorithms for solving several kinds of multi-objective optimization problems based on TOPSIS approach are presented in [3].

S. F. El-Feky and T.H.M. Abou-El-Enien, [4], develop a methodology to find compromise solutions for rough multiobjective optimization problems.

In the following section, the formulation of RMLMODM problems is given. A new hybrid algorithm based on the TOPSIS method [5-7], and "Lower \& Upper" approximations method [1-2], for solving RMLMODM problems is proposed in section (3). For the sake of illustration, we present an example and compared the solution of the example by the proposed algorithm with the ideal solutions and the solution of global criterion (GC) method.

\section{PROBLEM DEFINITION}

Consider the following linear multi-level multi-objective decision making (LMLMODM) problem with rough parameters in the objective functions:

$\left[\mathrm{DM}_{L_{1}}\right]$

$\underset{X_{1}}{\operatorname{Maximize}}\left(f_{11}\left(X_{1}, \ldots, X_{h}\right), \ldots, f_{1 k_{1}}\left(X_{1}, \ldots, X_{h}\right)\right)$ where, $X_{2}$ solves the $2^{\text {nd }}$ level

$\left[\mathrm{DM}_{L_{2}}\right]$

$\underset{X_{2}}{\operatorname{Maximize}}\left(f_{21}\left(X_{1}, \ldots, X_{h}\right), \ldots, f_{2 k_{2}}\left(X_{1}, \ldots, X_{h}\right)\right)$

where, $X_{3}$ solves the $3^{\text {rd }}$ level

$\left[\mathrm{DM}_{L_{3}}\right]$

$\underset{X_{3}}{\operatorname{Maximize}}\left(f_{31}\left(X_{1}, \ldots, X_{h}\right), \ldots, f_{3 k_{3}}\left(X_{1}, \ldots, X_{h}\right)\right)$

where, $X_{4}$ solves the $4^{\text {th }}$ level

where, $X_{I_{h}}$ solvesthe $h^{\text {th }}$ level

$\left[\mathrm{DM}_{L_{h}}\right]$

$\underset{X_{h}}{\operatorname{Maximize}}\left(f_{h 1}\left(X_{1}, \ldots, X_{h}\right), \ldots, f_{h k_{h}}\left(X_{1}, \ldots, X_{h}\right)\right)$

subject to

$X \in \mathbb{M}=\left\{X \in \mathbb{R}^{n}: D X \leq b\right\}$

where

$$
\begin{gathered}
f_{i q}=\sum_{j=1}^{n} c_{i q, j}\left(\left[Q_{i q, j}^{L L}, Q_{i q, j}^{H L}\right],\left[Q_{i q, j}^{L H}, Q_{i q, j}^{H H}\right]\right) x_{j}, i= \\
1,2, \ldots ., h, q=1,2, \ldots, k_{i},
\end{gathered}
$$

$m$ : The number of constraints, $n$ : The number of variables, $\mathrm{h}$ : The number of levels, 
$k$ : The number of objective functions,

$\mathrm{DM}_{L_{i}}: \mathrm{j}^{\text {th }}$ level decision maker, $\mathrm{i}=1,2, \ldots, \mathrm{h}$,

$k_{i}$ : The number of objective functions of the $\mathrm{DM}_{L_{i}}$, $\mathrm{i}=1,2, \ldots, \mathrm{h}$,

$K=K_{1} \cup K_{2} \cup \ldots \cup K_{j}=\{1,2, \ldots \ldots, k\}$,

$n_{i}$ : The number of variables of the $\mathrm{DM}_{L_{i}}, \mathrm{i}=1,2, \ldots, \mathrm{h}$,

$c_{i q, j}$ : Real constants coefficients of the objective functions , $\left(i=1,2, \ldots, h, q=1,2, \ldots, k_{i}, j=1, \ldots, n\right)$.

$b$ : An $m$-dimensional column vector of right-hand sides of constraints

$D:$ An $(m \times n)$ coefficient matrix,

$\mathbb{R}$ : The set of all real numbers,

$X$ : An $n$-dimensional column vector of variables,

$X_{i}$ : An $n_{i}$-dimensional column vector of variables for the $\mathrm{i}^{\text {th }}$ level, $\mathrm{i}=1,2, \ldots, \mathrm{h}$,

$\mathbb{N}=\{1,2, \ldots \ldots, n\}$,

$\mathbb{R}^{n}=\left\{X=\left(x_{1}, x_{2}, \ldots, x_{n}\right)^{T}: x_{i} \in \mathbb{R}, i \in \mathbb{N}\right\}$,

$\left[Q_{i q, j}^{L L}, Q_{i q, j}^{H L}\right],\left[Q_{i q, j}^{L H}, Q_{i q, j}^{H H}\right]$ are rough interval coefficients of the objective functions , $\left(i=1,2, \ldots, h, q=1,2, \ldots, k_{i}, j=\right.$ $1, \ldots, n)$.

Using the upper and lower approximation method, [2], the multi-level multi-objective decision making problems with rough parameters in the objective functions (RMLMODM) can be transformed to the following four deterministic linear multi-objective decision making (LMODM) problems :

$\underline{\boldsymbol{P}^{L L}}$ :(Lower interval coefficients-Lower interval)

$\left[\mathrm{DM}_{L_{1}}\right]$

$\underset{X_{1}}{\operatorname{Maximize}}\left(F_{11}^{L L}\left(X_{1}, \ldots, X_{h}\right), \ldots, F_{1 k_{1}}^{L L}\left(X_{1}, \ldots, X_{h}\right)\right)$

where, $X_{2}$ solves the $2^{\text {nd }}$ level

$\left[\mathrm{DM}_{L_{2}}\right]$

$\underset{X_{2}}{\operatorname{Maximize}}\left(F_{21}^{L L}\left(X_{1}, \ldots, X_{h}\right), \ldots, F_{2 k_{2}}^{L L}\left(X_{1}, \ldots, X_{h}\right)\right)$

where, $X_{3}$ solves the $3^{r d}$, level

$\left[\mathrm{DM}_{L_{3}}\right]$

$\underset{X_{3}}{\operatorname{Maximize}}\left(F_{31}^{L L}\left(X_{1}, \ldots, X_{h}\right), \ldots, F_{3 k_{3}}^{L L}\left(X_{1}, \ldots, X_{h}\right)\right)$

where, $X_{4}$ solves the $4^{\text {th }}$ level

where, $X_{I_{h}}$ solvesthe $h^{\text {th }}$ level

$\left[\mathrm{DM}_{L_{h}}\right]$

$\underset{X_{h}}{\operatorname{Maximize}}\left(F_{h 1}^{L L}\left(X_{1}, \ldots, X_{h}\right), \ldots, F_{h k_{h}}^{L L}\left(X_{1}, \ldots, X_{h}\right)\right)$

subject to

$X \in \mathbb{M}$
$\underline{P^{H L}}$ :(Upper interval coefficients-Lower interval)

$\left[\mathrm{DM}_{\mathrm{L}_{1}}\right]$

$\underset{\mathrm{X}_{1}}{\operatorname{Maximize}}\left(\mathrm{F}_{11}^{\mathrm{HL}}\left(\mathrm{X}_{1}, \ldots, \mathrm{X}_{\mathrm{h}}\right), \ldots, \mathrm{F}_{1 \mathrm{k}_{1}}^{\mathrm{HL}}\left(\mathrm{X}_{1}, \ldots, \mathrm{X}_{\mathrm{h}}\right)\right)$

where, $\mathrm{X}_{2}$ solves the $2^{\text {nd }}$ level

$\left[\mathrm{DM}_{\mathrm{L}_{2}}\right]$

$\underset{\mathrm{X}_{2}}{\operatorname{Maximize}}\left(\mathrm{F}_{21}^{\mathrm{HL}}\left(\mathrm{X}_{1}, \ldots, \mathrm{X}_{\mathrm{h}}\right), \ldots, \mathrm{F}_{2 \mathrm{k}_{2}}^{\mathrm{HL}}\left(\mathrm{X}_{1}, \ldots, \mathrm{X}_{\mathrm{h}}\right)\right)$

where, $\mathrm{X}_{3}$ solves the $3^{\text {rd }}$ level

$\left[\mathrm{DM}_{\mathrm{L}_{3}}\right]$

$\underset{\mathrm{X}_{3}}{\operatorname{Maximize}}\left(\mathrm{F}_{31}^{\mathrm{HL}}\left(\mathrm{X}_{1}, \ldots, \mathrm{X}_{\mathrm{h}}\right), \ldots, \mathrm{F}_{3 \mathrm{k}_{3}}^{\mathrm{HL}}\left(\mathrm{X}_{1}, \ldots, \mathrm{X}_{\mathrm{h}}\right)\right)$

where, $\mathrm{X}_{4}$ solves the $4^{\text {th }}$ level

where, $\mathrm{X}_{\mathrm{I}_{\mathrm{h}}}$ solves the $\mathrm{h}^{\text {th }}$ level

$\left[\mathrm{DM}_{\mathrm{L}_{\mathrm{h}}}\right]$

$\underset{\mathrm{X}_{\mathrm{h}}}{\operatorname{Maximize}}\left(\mathrm{F}_{\mathrm{h} 1}^{\mathrm{HL}}\left(\mathrm{X}_{1}, \ldots, \mathrm{X}_{\mathrm{h}}\right), \ldots, \mathrm{F}_{\mathrm{hk}}^{\mathrm{HL}}\left(\mathrm{X}_{1}, \ldots, \mathrm{X}_{\mathrm{h}}\right)\right)$

subject to

$\mathrm{X} \in \mathbb{M}$

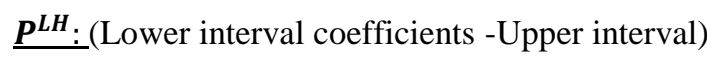

$\left[\mathrm{DM}_{L_{1}}\right]$

$\underset{X_{1}}{\operatorname{Maximize}}\left(F_{11}^{L H}\left(X_{1}, \ldots, X_{h}\right), \ldots, F_{1 k_{1}}^{L H}\left(X_{1}, \ldots, X_{h}\right)\right)$

where, $X_{2}$ solves the $2^{\text {nd }}$ level

$\left[\mathrm{DM}_{L_{2}}\right]$

$\underset{X_{2}}{\operatorname{Maximize}}\left(F_{21}^{L H}\left(X_{1}, \ldots, X_{h}\right), \ldots, F_{2 k_{2}}^{L H}\left(X_{1}, \ldots, X_{h}\right)\right)$

where, $X_{3}$ solves the $3^{\text {rd }}$ level

$\left[\mathrm{DM}_{L_{3}}\right]$

$\underset{X_{3}}{\operatorname{Maximize}}\left(F_{31}^{L H}\left(X_{1}, \ldots, X_{h}\right), \ldots, F_{3 k_{3}}^{L H}\left(X_{1}, \ldots, X_{h}\right)\right)$

where, $X_{4}$ solves the $4^{\text {th }}$ level

where, $X_{I_{h}}$ solves the $h^{\text {th }}$ level

$\left[\mathrm{DM}_{L_{h}}\right]$

$\underset{X_{h}}{\operatorname{Maximize}}\left(F_{h 1}^{L H}\left(X_{1}, \ldots, X_{h}\right), \ldots, F_{h k_{h}}^{L H}\left(X_{1}, \ldots, X_{h}\right)\right)$ 
subject to

$X \in \mathbb{M}$

$\underline{\boldsymbol{P}^{H \boldsymbol{H}}}$ :(Upper interval coefficients -Upper interval)

$\left[\mathrm{DM}_{\mathrm{L}_{1}}\right]$

$\underset{\mathrm{X}_{1}}{\operatorname{Maximize}}\left(\mathrm{F}_{11}^{\mathrm{HH}}\left(\mathrm{X}_{1}, \ldots, \mathrm{X}_{\mathrm{h}}\right), \ldots, \mathrm{F}_{1 \mathrm{k}_{1}}^{\mathrm{HH}}\left(\mathrm{X}_{1}, \ldots, \mathrm{X}_{\mathrm{h}}\right)\right)$

where, $\mathrm{X}_{2}$ solves the $2^{\text {nd }}$ level

$\left[\mathrm{DM}_{\mathrm{L}_{2}}\right]$

$\underset{\mathrm{X}_{2}}{\operatorname{Maximize}}\left(\mathrm{F}_{21}^{\mathrm{HH}}\left(\mathrm{X}_{1}, \ldots, \mathrm{X}_{\mathrm{h}}\right), \ldots, \mathrm{F}_{2 \mathrm{k}_{2}}^{\mathrm{HH}}\left(\mathrm{X}_{1}, \ldots, \mathrm{X}_{\mathrm{h}}\right)\right)$

where, $\mathrm{X}_{3}$ solves the $3^{\text {rd }}$ level

$\left[\mathrm{DM}_{\mathrm{L}_{3}}\right]$

$\underset{\mathrm{X}_{3}}{\operatorname{Maximize}}\left(\mathrm{F}_{31}^{\mathrm{HH}}\left(\mathrm{X}_{1}, \ldots, \mathrm{X}_{\mathrm{h}}\right), \ldots, \mathrm{F}_{3 \mathrm{k}_{3}}^{\mathrm{HH}}\left(\mathrm{X}_{1}, \ldots, \mathrm{X}_{\mathrm{h}}\right)\right)$

where, $\mathrm{X}_{4}$ solves the $4^{\text {th }}$ level

where, $\mathrm{X}_{\mathrm{I}_{\mathrm{h}}}$ solvesthe $\mathrm{h}^{\text {th }}$ level

$\left[\mathrm{DM}_{\mathrm{L}_{\mathrm{h}}}\right]$

$\underset{\mathrm{X}_{\mathrm{h}}}{\operatorname{Maximize}}\left(\mathrm{F}_{\mathrm{h} 1}^{\mathrm{HH}}\left(\mathrm{X}_{1}, \ldots, \mathrm{X}_{\mathrm{h}}\right), \ldots, \mathrm{F}_{\mathrm{hk}}^{\mathrm{HH}}\left(\mathrm{X}_{1}, \ldots, \mathrm{X}_{\mathrm{h}}\right)\right)$

subject to

$\mathrm{X} \in \mathbb{M}$

where

$F_{i q}^{L L}=\sum_{j=1}^{n} c_{i q, j} Q_{i q, j}^{L L} x_{j}, i=1,2, \ldots, h, q=1,2, \ldots, k_{i}$,

$F_{i q}^{L H}=\sum_{j=1}^{n} c_{i q, j} Q_{i q, j}^{L H} x_{j}, i=1,2, \ldots, h, q=1,2, \ldots, k_{i}$

$F_{i q}^{H L}=\sum_{j=1}^{n} c_{i q, j} Q_{i q, j}^{H L} x_{j}, i=1,2, \ldots, h, q=1,2, \ldots, k_{i}$,

$F_{i q}^{H H}=\sum_{j=1}^{n} c_{i q, j} Q_{i q, j}^{H H} x_{j}, i=1,2, \ldots, h, q=1,2, \ldots, k_{i}$,

\section{HYBRID ALGORITHM FOR RMLMODEM}

A modified version of TOPSIS method, [3, 5], is introduced to find compromise solutions, [8-10], for the RMLMODM problems. Modified equations for the distance function equation from the positive ideal solution (PIS) and the distance function equation from the negative ideal solution (NIS) are introduced. Thus, we present the following hybrid algorithm based on a modified version of TOPSIS method and the upper and lower approximation method to generate compromise solutions, [4], for RMLMODM problems.

Algorithm:

Phase (1):

Step 1:
Let $\mathrm{h}=$ the number of the levels of the RMLMODM problem (1). Set $\mathrm{i}=1$, "The $1^{\text {st }}$ level".

Step 2:

Use the "Lower \& Upper" approximations method to transform the RMLMODM Problem (1) into the four deterministic LMLMODM problems (3)-(6).

Step 3:

Construct the positive ideal solution (PIS) payoff tables, [4], of the following problems, for $\mathrm{i}=1$ :

$\left[\mathrm{DM}_{L_{i}}\right]$

$\underset{X_{i}}{\operatorname{Maximize}}\left(F_{i 1}^{L L}\left(X_{1}, \ldots, X_{h}\right), \ldots, F_{i k_{i}}^{L L}\left(X_{1}, \ldots, X_{h}\right)\right)$

subject to

$X \in \mathbb{M}$,

$\underset{X_{i}}{\operatorname{Maximize}}\left(F_{i 1}^{H L}\left(X_{1}, \ldots, X_{h}\right), \ldots, F_{i k_{i}}^{H L}\left(X_{1}, \ldots, X_{h}\right)\right)$

subject to

$X \in \mathbb{M}$,

$\underset{X_{i}}{\operatorname{Maximize}}\left(F_{i 1}^{L H}\left(X_{1}, \ldots, X_{h}\right), \ldots, F_{i k_{i}}^{L H}\left(X_{1}, \ldots, X_{h}\right)\right)$

subject to

$X \in \mathbb{M}$,

$\underset{X_{i}}{\operatorname{Maximize}}\left(F_{i 1}^{H H}\left(X_{1}, \ldots, X_{h}\right), \ldots, F_{i k_{i}}^{H H}\left(X_{1}, \ldots, X_{h}\right)\right)$

subject to

$X \in \mathbb{M}$,

and obtain the PIS:

$\left(F_{1 q}^{*}{ }^{\mathrm{LL}-\mathrm{DM}_{L_{1}}}\right),\left(F_{1 q}^{*}{ }^{\mathrm{HL}-\mathrm{DM}_{L_{1}}}\right),\left(F_{1 q}^{*}{ }^{\mathrm{L} H-\mathrm{DM}_{L_{1}}}\right)$ and $\left(F_{1 q}^{*}{ }^{\mathrm{HH}-\mathrm{DM}_{L_{1}}}\right)$,
$q=1,2, \ldots, k_{1}$.

Step 4:

Construct the negative ideal solution (NIS) payoff tables, [4], of the problems (11-14) for $\mathrm{i}=1$ and obtain the NIS: $\left(F_{1 q}^{-{ }^{L L-D M_{L_{1}}}}\right),\left(F_{1 q}^{-{ }^{\mathrm{HL}-\mathrm{D} M_{L_{1}}}}\right),\left(F_{1 q}^{-{ }^{\mathrm{LH}-\mathrm{D} M_{L_{1}}}}\right)$ and $\left(F_{1 q}^{-{ }^{\mathrm{HH}-D M_{L_{1}}}}\right)$, $q=1,2, \ldots, k_{1}$.

Step 5:

Let $w_{1 q}=w_{1 q}{ }^{*}$ where $\sum_{q=1}^{k_{1}} w_{1 q}=1$ and $p=p^{*}, p^{*} \in$ $\{1,2, \ldots, \infty\}$.

Step 6:

Construct distance functions $d_{p}^{P I S^{\mathrm{LL}-D M_{L_{1}}}}$ and $d_{p}^{N I S^{\mathrm{LL}-\mathrm{D} M_{L_{1}}}}$, [4], by the above steps (3, $4 \& 5)$ :

$d_{p}^{P I S^{L L-D M_{L_{1}}}}=$ 


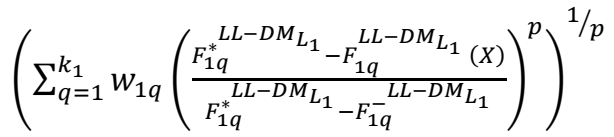

And

$d_{p}^{N I S^{L L-D M_{L_{1}}}}=$

$\left(\sum_{q=1}^{k_{1}} w_{1 q}\left(\frac{F_{1 q}^{L L-D M_{L_{1}}}(X)-F_{1 q}^{-L L-D M_{L_{1}}}}{F_{1 q}^{* L-D M_{L_{1}}-F_{1 q}^{-}{ }^{L L-D M_{L_{1}}}}}\right)^{p}\right)^{1 / p}$

Construct distance functions $d_{p}^{P I S^{\mathrm{HL}-D M_{L_{1}}}}$ and $d_{p}^{N I S^{\mathrm{HL}-\mathrm{D} M_{L_{1}}}}$ by the above steps $(3,4 \& 5)$ :

$d_{p}^{P I S^{\mathrm{HL}-D M_{L_{1}}}}=$

$\left(\sum_{q=1}^{k_{1}} w_{1 q}\left(\frac{F_{1 q}^{*}{ }^{H L-D M_{L_{1}}-F_{1 q}}{ }^{H L-D M_{L_{1}}}(X)}{F_{1 q}^{*}{ }^{H L-D M_{L_{1}}-F_{1 q}^{-}}{ }^{H L-D M_{L_{1}}}}\right)^{p}\right)^{1 / p}$

and

$d_{p}^{N I S^{H L-D M_{L_{1}}}}=$

$\left(\sum_{q=1}^{k_{1}} W_{1 q}\left(\frac{F_{1 q}^{H L-D M_{L_{1}}}(X)-F_{1 q}^{-}{ }^{H L-D M_{L_{1}}}}{F_{1 q}^{*}{ }^{H L-D M_{L_{1}}-F_{1 q}^{-}}{ }^{H L-D M_{L_{1}}}}\right)^{p}\right)^{1 / p}$

Construct distance functions $d_{p}^{P I S^{\mathrm{L} H-D M_{L_{1}}}}$ and $d_{p}^{N I S^{\mathrm{LH}-\mathrm{D} M_{L_{1}}}}$ by the above steps $(3,4 \& 5)$ :

$d_{p}^{P I S^{L H-D M_{L_{1}}}}=$

$\left(\sum_{q=1}^{k_{1}} w_{1 q}\left(\frac{F_{1 q}^{*}{ }^{L H-D M_{L_{1}}}-F_{1 q}^{L H-D M_{L_{1}}}(X)}{F_{1 q}^{*}{ }^{L H-D M_{L_{1}}}-F_{1 q}^{-}{ }^{L H-D M_{L_{1}}}}\right)^{p}\right)^{1 / p}$

and

$d_{p}^{N I S^{L H-D M_{L_{1}}}}=$

$\left(\sum_{q=1}^{k_{1}} w_{1 q}\left(\frac{F_{1 q}^{L H-D M_{L_{1}}}(X)-F_{1 q}^{-{ }^{L H-D M_{L_{1}}}}}{F_{1 q}^{{ }^{L H-D M_{L_{1}}}-F_{1 q}^{-}{ }^{L H-D M_{L_{1}}}}}\right)^{p}\right)^{1 / p}$

Construct distance functions $d_{p}^{P I S^{\mathrm{HH}-D M_{L_{1}}}}$ and $d_{p}^{N I S^{\mathrm{HH}-\mathrm{D} M_{L_{1}}}}$ by the above steps $(3,4 \& 5)$ :

$d_{p}^{P I S}{ }^{\mathrm{HH}-D M_{L_{1}}}=$

$\left(\sum_{q=1}^{k_{1}} W_{1 q}\left(\frac{F_{1 q}^{*}{ }^{H H-D M_{L_{1}}-F_{1 q} H H-D M_{L_{1}}}(X)}{F_{1 q}^{*}{ }^{H H-D M_{L_{1}}}-F_{1 q}^{-}{ }^{-H H-D M_{L_{1}}}}\right)^{p}\right)^{1 / p}$

and

$d_{p}^{N I S^{H H-D M_{L_{1}}}}=$ $\left(\sum_{q=1}^{k_{1}} w_{1 q}\left(\frac{F_{1 q}^{H H-D M_{L_{1}}}(X)-F_{1 q}^{-H H-D M_{L_{1}}}}{F_{1 q}^{* H H-D M_{L_{1}} F_{1 q}^{-}{ }^{H H-D M_{L_{1}}}}}\right)^{p}\right)^{1 / p}$

\section{Step 7:}

Construct the following bi-objective problem with two commensurable (but conflicting) objectives, [4], using the distance functions $d_{p}^{P I S^{\mathrm{LL}-D M_{L_{1}}}}$ and $d_{p}^{N I S^{\mathrm{LL}-\mathrm{D} M_{L_{1}}}}$ :

Minimize $d_{p}^{P I S^{L L-D M_{L_{1}}}}(X)$

Maximize $d_{p}^{N I S^{\mathrm{LL}-\mathrm{D} M_{L_{1}}}}(X)$

subject to

\section{$X \in \mathbb{M}$}

where

$p=1,2, \ldots \ldots, \infty$.

Construct the following bi-objective problem with two commensurable (but conflicting) objectives using the distance

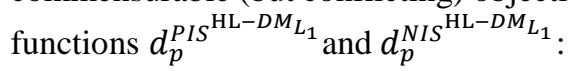

Minimize $d_{p}^{P I S^{\mathrm{HL}-D M_{L_{1}}}}(X)$

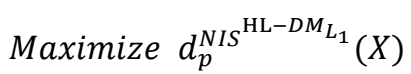

subject to

$X \in \mathbb{M}$

where

$p=1,2, \ldots \ldots, \infty$.

Construct the following bi-objective problem with two commensurable (but conflicting) objectives using the distance functions $d_{p}^{P I S^{\mathrm{LH}-D M_{L_{1}}}}$ and $d_{p}^{N I S^{\mathrm{LH}-D M_{L_{1}}}}$ :

Minimize $d_{p}^{\text {PIS }}{ }^{\mathrm{LH}-D M_{L_{1}}}(X)$

Maximize $d_{p}^{N I S^{\mathrm{LH}-D M_{L_{1}}}}(X)$

subject to

$X \in \mathbb{M}$

where

$p=1,2, \ldots \ldots, \infty$.

Construct the following bi-objective problem with two commensurable (but conflicting) objectives using the distance

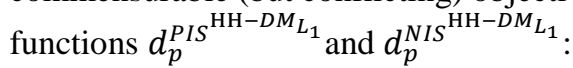

Minimized ${ }_{p}^{P I S^{\mathrm{HH}-D M_{L_{1}}}}(X)$ 


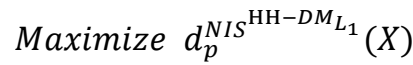

subject to

$\mathrm{X} \in \mathbb{M}$

where

$p=1,2, \ldots \ldots, \infty$.

\section{Step 8:}

Construct PIS Payoff table for problem (23) and obtain:

$d_{p}^{-L L-D M_{L_{1}}}=\left(\left(d_{p}^{P I S^{L L-D M_{L_{1}}}}\right)^{-},\left(d_{p}^{N I S^{L L-D M_{L_{1}}}}\right)^{-}\right)$,

$d_{p}^{* \mathrm{LL}-D M_{L_{1}}}=\left(\left(d_{p}^{P I S^{\mathrm{LL}-D M_{L_{1}}}}\right)^{*},\left(d_{p}^{N I S^{\mathrm{LL}-D M_{L_{1}}}}\right)^{*}\right)$.

where

$\left(d_{p}^{P I S^{L L-D M_{L_{1}}}}\right)^{*}$

$=\underset{X \in \mathbb{M}}{\text { Minimize }} d_{p}^{P I S}{ }^{L L-D M_{L_{1}}}(X)$ and the solution is $X^{P I S^{L L-D M L_{1}}}$,

$\left(d_{p}^{N I S^{L L-D M_{L_{1}}}}\right)^{*}$

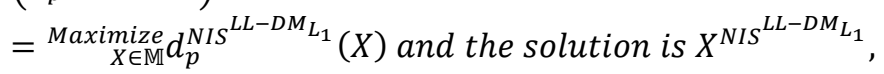

$\left(d_{p}^{P I S^{L L-D M_{L_{1}}}}\right)^{-}=d_{p}^{P I S^{L L-D M_{L_{1}}}}\left(X^{N I S^{L L-D M_{L_{1}}}}\right)$ and

$\left(d_{p}^{N I S^{L L-D M_{L_{1}}}}\right)^{-}=d_{p}^{N I S^{L L-D M_{L_{1}}}}\left(X^{P I S^{L L-D M_{L_{1}}}}\right)$.

Construct PIS Payoff table for problem (24) and obtain:

$\mathrm{d}_{\mathrm{p}}^{-\mathrm{HL}^{-\mathrm{DM}_{\mathrm{L}_{1}}}}=\left(\left(\mathrm{d}_{\mathrm{p}}^{\mathrm{PIS}}{ }^{\mathrm{HL}-\mathrm{DM} \mathrm{L}_{1}}\right)^{-},\left(\mathrm{d}_{\mathrm{p}}^{\mathrm{NIS}}{ }^{\mathrm{HL}-\mathrm{DM}_{\mathrm{L}_{1}}}\right)^{-}\right)$,

$\mathrm{d}_{\mathrm{p}}^{\mathrm{HL}^{\mathrm{HL}-\mathrm{DM}_{\mathrm{L}_{1}}}}=\left(\left(\mathrm{d}_{\mathrm{p}}^{\mathrm{PIS}}{ }^{\mathrm{HL}-\mathrm{DM} \mathrm{L}_{1}}\right)^{*},\left(\mathrm{~d}_{\mathrm{p}}^{\mathrm{NIS}}{ }^{\mathrm{HL}-\mathrm{DM} \mathrm{L}_{1}}\right)^{*}\right)$.

Construct PIS Payoff table for problem (21) and obtain:

$\mathrm{d}_{\mathrm{p}}^{\mathrm{L}^{\mathrm{LH}-\mathrm{DM}_{\mathrm{L}_{1}}}}=\left(\left(\mathrm{d}_{\mathrm{p}}^{\mathrm{PIS} \mathrm{L}^{\mathrm{LH}-\mathrm{DM}_{\mathrm{L}_{1}}}}\right)^{-},\left(\mathrm{d}_{\mathrm{p}}^{\mathrm{NIS}}{ }^{\mathrm{LH}-\mathrm{DM}_{\mathrm{L}_{1}}}\right)^{-}\right)$,

$\mathrm{d}_{\mathrm{p}}^{* \mathrm{LH}^{\mathrm{DH} \mathrm{L}_{\mathrm{L}_{1}}}}=\left(\left(\mathrm{d}_{\mathrm{p}}^{\mathrm{PI}}{ }^{\mathrm{LH}-\mathrm{DM} \mathrm{L}_{1}}\right)^{*},\left(\mathrm{~d}_{\mathrm{p}}^{\mathrm{NI}}{ }^{\mathrm{LH}-\mathrm{DM}_{\mathrm{L}_{1}}}\right)^{*}\right)$.

Construct PIS Payoff table for problem (22) and obtain:

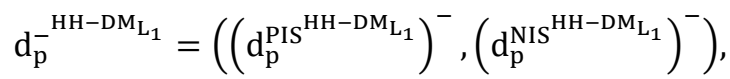

$\mathrm{d}_{\mathrm{p}}^{* \mathrm{HH}-\mathrm{DM}_{\mathrm{L}_{1}}}=\left(\left(\mathrm{d}_{\mathrm{p}}^{\mathrm{PIS}}{ }^{\mathrm{HH}-\mathrm{DM}_{\mathrm{L}_{1}}}\right)^{*},\left(\mathrm{~d}_{\mathrm{p}}^{\mathrm{NIS}}{ }^{\mathrm{HH}-\mathrm{DM}_{\mathrm{L}_{1}}}\right)^{*}\right)$

$\underline{\text { Step 9: }}$

Construct the following satisfactory level model, [11-13], (for finite value of $\mathrm{p}$ ) for problem (23):

Maximize $\delta^{\mathrm{LL}^{\mathrm{D}} \mathrm{DM}_{\mathrm{L}_{1}}}$ subject to

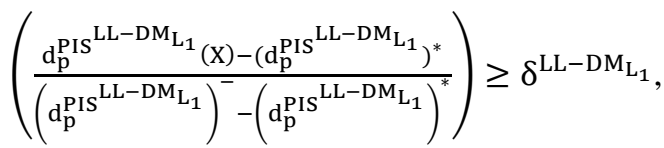

$\left(\frac{\left(\mathrm{d}_{\mathbf{p}}^{\mathrm{NIS}}{ }^{\mathrm{LL}-\mathrm{DM}_{\mathrm{L}_{1}}}\right)^{*}-\mathrm{d}_{\mathrm{p}}^{\mathrm{NIS}}{ }^{\mathrm{LL}-\mathrm{DM}_{\mathrm{L}_{1}}}(\mathrm{X})}{\left(\mathrm{d}_{\mathbf{p}}^{\mathrm{NIS}}{ }^{\mathrm{LL}-\mathrm{DM}_{\mathrm{L}_{1}}}\right)^{*}-\left(\mathrm{d}_{\mathbf{p}}^{\mathrm{NIS}}{ }^{\mathrm{LL}-\mathrm{DM}_{\mathrm{L}_{1}}}\right)}\right) \geq \delta^{\mathrm{LL}-\mathrm{DM}_{\mathrm{L}_{1}}}$,

$\mathrm{X} \in \mathbb{M}, \delta^{\mathrm{LL}-\mathrm{DM}_{\mathrm{L}_{1}}} \in[0,1]$

Construct the following satisfactory level model (for finite value of $p$ ) for problem (24):

Maximize $\delta^{\mathrm{HL}-\mathrm{DM}_{\mathrm{L}_{1}}}$

subject to

$\left(\frac{d_{p}^{\mathrm{PIS}}{ }^{\mathrm{HL}-\mathrm{DM} \mathrm{L}_{\mathrm{L}_{1}}}(\mathrm{X})-\left(\mathrm{d}_{\mathrm{p}}^{\mathrm{PIS}}{ }^{\mathrm{HL}-\mathrm{DM} \mathrm{L}_{\mathrm{L}_{1}}}\right)^{*}}{\left(\mathrm{~d}_{\mathrm{p}}^{\mathrm{PIS}}{ }^{\mathrm{HL}-\mathrm{DM} \mathrm{L}_{\mathrm{L}_{1}}}\right)^{-}-\left(\mathrm{d}_{\mathrm{p}}^{\mathrm{PIS}}{ }^{\mathrm{HL}-\mathrm{DM}_{\mathrm{L}_{1}}}\right)^{*}}\right) \geq \delta^{\mathrm{HL}-\mathrm{DM}_{\mathrm{L}_{1}}}$,

$\left(\frac{\left(\mathrm{d}_{\mathbf{p}}^{\mathrm{NIS}}{ }^{\mathrm{HL}-\mathrm{DM}_{\mathrm{L}_{1}}}\right)^{*}-\mathrm{d}_{\mathrm{p}}^{\mathrm{NIS}}{ }^{\mathrm{HL}-\mathrm{DM}_{\mathrm{L}_{1}}}(\mathrm{X})}{\left(\mathrm{d}_{\mathbf{p}}^{\mathrm{NIS}}{ }^{\mathrm{HL}-\mathrm{DM}_{\mathrm{L}_{1}}}\right)^{*}-\left(\mathrm{d}_{\mathbf{p}}^{\mathrm{NIS}}{ }^{\mathrm{HL}-\mathrm{DM}_{\mathrm{L}_{1}}}\right)^{-}}\right) \geq \delta^{\mathrm{HL}-\mathrm{DM}_{\mathrm{L}_{1}}}$

$\mathrm{X} \in \mathbb{M}, \delta^{\mathrm{HL}-\mathrm{DM}_{\mathrm{L}_{1}}} \in[0,1]$

Construct the following satisfactory level model (for finite value of $p$ ) for problem (25):

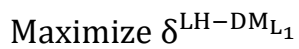

subject to

$\left(\frac{d_{p}^{P I S^{L H-D M_{L_{1}}}}(X)-\left(d_{p}^{P I S^{L H-D M_{L_{1}}}}\right)^{*}}{\left(d_{p}^{P I S^{L H-D M_{L_{1}}}}\right)^{-}-\left(d_{p}^{P I S^{L H-D M_{L_{1}}}}\right)^{*}}\right) \geq \delta^{L H-D M_{L_{1}}}$

$\left(\frac{\left(d_{p}^{N I S^{L H-D M_{L_{1}}}}\right)^{*}-d_{p}^{N I S^{L H-D M_{L_{1}}}(X)}}{\left(d_{p}^{N I S^{L H-D M_{L_{1}}}}\right)^{*}-\left(d_{p}^{N I S^{L H-D M_{L_{1}}}}\right)^{-}}\right) \geq \delta^{L H-D M_{L_{1}}}$,

$X \in \mathbb{M}, \delta^{L H-D M_{L_{1}}} \in[0,1]$

Construct the following satisfactory level model (for finite value of $p$ ) for problem (26):

Maximize $\delta^{H H-D M_{L_{1}}}$

subject to

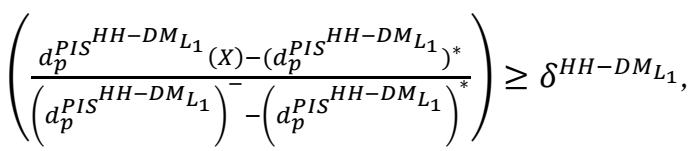


$\left(\frac{\left(d_{p}^{N I S^{H H-D M_{L_{1}}}}\right)^{*}-d_{p}^{N I S^{H H-D M_{L_{1}}}(X)}}{\left(d_{p}^{N I S^{H H-D M_{L_{1}}}}\right)^{*}-\left(d_{p}^{N I S^{H H-D M_{L_{1}}}}\right)^{-}}\right) \geq \delta^{H H-D M_{L_{1}}}$,

$\mathrm{X} \in \mathbb{M}, \delta^{\mathrm{HH}-\mathrm{DM}_{\mathrm{L}_{1}}} \in[0,1]$

\section{$\underline{\text { Step 10: }}$}

Solve problems (27-30) to obtain the satisfactory levels $\delta^{*^{\mathrm{LL}-D M_{L_{1}}}}, \delta^{*^{\mathrm{HL}-D M_{L_{1}}}}, \delta^{*^{\mathrm{LH}-D M_{L_{1}}}}$ and $\delta^{*^{\mathrm{HH}-D M_{L_{1}}}}$ for the compromise solutions $X^{*}{ }^{\mathrm{LL}-D M_{L_{1}}}, X^{*^{\mathrm{HL}-D M_{L_{1}}}}, X^{*}{ }^{\mathrm{LH}-D M_{L_{1}}}$ and $X^{*}{ }^{\mathrm{HH}-D M_{L_{1}}}$. If the $\mathrm{DM}_{L_{1}}$ is satisfied with the solutions, then go to step (11). Otherwise, go to step (5).

\section{Step 11:}

Ask the $\mathrm{DM}_{\mathrm{L}_{1}}$ to select the maximum acceptable negative and positive tolerance (relaxation) values, [14, 15]: $\tau_{i}^{L L-D M_{L_{1}}-\mathrm{L}}, \tau_{i}^{L L-D M_{L_{1}}-R}, \tau_{i}^{H L-D M_{L_{1}}-L}, \tau_{i}^{H L-D M_{L_{1}}-R}, \tau_{i}^{L H-D M_{L_{1}}-L}$ $\tau_{\mathrm{i}}^{\mathrm{HH}-\mathrm{DM}_{\mathrm{L}_{1}}-\mathrm{L}}$ and $\tau_{\mathrm{i}}^{\mathrm{HH}-\mathrm{DM}_{\mathrm{L}_{1}}-\mathrm{R}}$,

$i=1,2, \ldots, n_{1}$ on the decision vectors:

$$
\begin{gathered}
X_{1}^{*}{ }^{\mathrm{LL}-D M_{L_{1}}}= \\
\left(x_{11}^{* \mathrm{LL}-D M_{L_{1}}}, x_{12}^{* \mathrm{LL}-D M_{L_{1}}}, \ldots, x_{1 n_{1}}^{* \mathrm{LL}-D M_{L_{1}}}\right), X_{1}^{*}{ }^{\mathrm{HL}-D M_{L_{1}}}= \\
\left(x_{11}^{* \mathrm{HL}-D M_{L_{1}}}, x_{12}^{* \mathrm{HL}-D M_{L_{1}}}, \ldots, x_{1 n_{1}}^{* \mathrm{HL}-D M_{L_{1}}}\right),
\end{gathered}
$$$$
X_{1}^{*}{ }^{\mathrm{LH}-D M_{L_{1}}}=\left(x_{11}^{*}{ }^{\mathrm{LH}-D M_{L_{1}}}, x_{12}^{*}{ }^{\mathrm{LH}-D M_{L_{1}}}, \ldots, x_{1 n_{1}}^{* \mathrm{LH}-D M_{L_{1}}}\right),
$$

and

$$
X_{1}^{*}{ }^{\mathrm{HH}-D M_{L_{1}}}=\left(x_{11}^{*}{ }^{\mathrm{HH}-D M_{L_{1}}}, x_{12}^{*}{ }^{\mathrm{HH}-D M_{L_{1}}}, \ldots, x_{1 n_{1}}^{* \mathrm{HH}-D M_{L_{1}}}\right) .
$$

Set $\mathrm{i}=\mathrm{i}+1$, go to the next phase.

\section{Phase (2):}

Step 12:

Set $\mathrm{i}=2$, "The $2^{\text {nd }}$ level".

Construct the PIS payoff table of problems (11-14) for $\mathrm{i}=2$, and obtain the PIS: $\left(F_{2 q}^{*}{ }^{\mathrm{LL}-\mathrm{DM}_{L_{2}}}\right),\left(F_{2 q}^{*}{ }^{\mathrm{HL}-\mathrm{DM}_{L_{2}}}\right),\left(F_{2 q}^{*}{ }^{\mathrm{LH}-\mathrm{DM}_{L_{2}}}\right)$ and $\left(F_{2 q}^{*}{ }^{\mathrm{HH}-\mathrm{DM}_{L_{2}}}\right), q=1,2, \ldots, k_{2}$.

\section{Step 13:}

Construct the negative ideal solution (NIS) payoff tables of the problems (11-14) for $\mathrm{i}=2$ and obtain the NIS: $\left(F_{2 q}^{-{ }^{L L-D M_{L_{2}}}}\right),\left(F_{2 q}^{{ }^{-\mathrm{HL}-\mathrm{D} M_{L_{2}}}}\right),\left(F_{2 q}^{{ }^{-\mathrm{LH}-\mathrm{D} M_{L_{2}}}}\right)$ and $\left(F_{2 q}^{{ }^{-\mathrm{HH}-D M_{L_{2}}}}\right)$, $q=1,2, \ldots, k_{2}$.

Step 14:

Let $w_{2 q}=w_{2 q}{ }^{*}$ where $\sum_{q=1}^{k_{1}} w_{2 q}=1$ and $p=p^{*}, p^{*} \in$ $\{1,2, \ldots, \infty\}$.

Step 15:

Construct distance functions $d_{p}^{P I S^{\mathrm{LL}-D M_{L_{2}}}}$ and $d_{p}^{N I S^{\mathrm{LL}-\mathrm{D} M_{L_{2}}}}$ :

$$
\begin{gathered}
d_{p}^{P I S^{L L-D M_{L_{2}}}}=\left(\sum_{q=1}^{k_{1}} W_{1 q}\left(\frac{F_{1 q}^{* L L-D M_{L_{1}}}-F_{1 q}^{L L-D M_{L_{1}}}(X)}{F_{1 q}^{* L L-D M_{L_{1}}}-F_{1 q}^{-L L-D M_{L_{1}}}}\right)^{p}+\right. \\
\left.\sum_{q=1}^{k_{2}} w_{2 q}\left(\frac{F_{2 q}^{*}{ }^{L L-D M_{L_{2}}}-F_{2 q}^{L L-D M_{L_{2}}}(X)}{F_{2 q}^{*}{ }^{L L-D M_{L_{1}}}-F_{2 q}^{-}{ }^{L L-D M_{L_{2}}}}\right)^{p}\right)^{1 / p}
\end{gathered}
$$

And

$$
\begin{gathered}
d_{p}^{N I S^{L L-D M_{L_{2}}}}=\left(\sum_{q=1}^{k_{1}} w_{1 q}\left(\frac{F_{1 q}^{L L-D M_{L_{1}}}(X)-F_{1 q}^{-}{ }^{L L-D M_{L_{1}}}}{F_{1 q}^{*}{ }^{L L M_{L_{1}}}-F_{1 q}^{-}{ }^{L L-D M_{L_{1}}}}\right)^{p}+\right. \\
\left.\sum_{q=2}^{k_{2}} w_{2 q}\left(\frac{F_{2 q}^{L L-D M_{L_{2}}}(X)-F_{2 q}^{-}{ }^{L L-D M_{L_{2}}}}{F_{2 q}^{*}{ }^{L L-D M_{L}}-F_{2 q}^{-}{ }^{L L-D M_{L_{2}}}}\right)^{1 / p}\right)^{1 / p}
\end{gathered}
$$

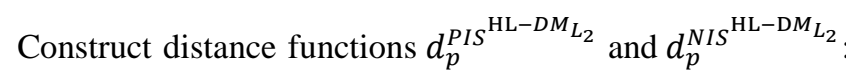

and

$$
\begin{aligned}
& d_{p}^{N I S^{H L-D M_{L_{2}}}}=
\end{aligned}
$$

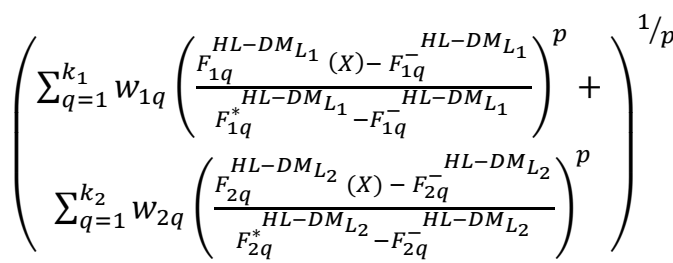

Construct distance functions $d_{p}^{P I S^{\mathrm{L} H-D M_{L_{2}}}}$ and $d_{p}^{N I S^{\mathrm{LH}-\mathrm{D} M_{L_{2}}}}$

$$
\begin{gathered}
d_{p}^{P I S^{L H-D M_{L_{2}}}}=\left(\sum_{q=1}^{k_{1}} W_{1 q}\left(\frac{F_{1 q}^{*}{ }^{L H-D M_{L_{1}}}-F_{1 q}^{L H-D M_{L_{1}}}(X)}{F_{1 q}^{*}{ }^{L H-D M_{L_{1}}}-F_{1 q}^{-}{ }^{L H-D M_{L_{1}}}}\right)^{p}+\right. \\
\left.\sum_{q=1}^{k_{2}} w_{2 q}\left(\frac{F_{2 q}^{{ }^{*}}{ }^{L H-D M_{L_{2}}}-F_{2 q}^{L H-D M_{L_{2}}}(X)}{F_{2 q}^{*}{ }^{L H-D M_{L_{2}}}-F_{2 q}^{-}{ }^{L H-D M_{L_{2}}}}\right)^{p}\right)^{1 / p}
\end{gathered}
$$

and

$$
\begin{gathered}
d_{p}^{N I S^{L H-D M_{L_{2}}}}=\left(\sum_{q=1}^{k_{1}} W_{1 q}\left(\frac{F_{1 q}^{L H-D M_{L_{1}}}(X)-F_{1 q}^{-L H-D M_{L_{1}}}}{F_{1 q}^{* L H-D M_{L_{1}}}-F_{1 q}^{-}{ }^{L H-D M_{L_{1}}}}\right)^{p}+\right. \\
\left.\sum_{q=1}^{k_{2}} W_{2 q}\left(\frac{F_{2 q}^{L H-D M_{L_{2}}}(X)-F_{2 q}^{-}{ }^{L H-D M_{L_{2}}}}{F_{2 q}^{*}{ }^{L H-D M_{L_{2}}}-F_{2 q}^{-L H-D M_{L_{2}}}}\right)^{p}\right)^{1 / p}
\end{gathered}
$$

Construct distance functions $d_{p}^{P I S^{\mathrm{HH}-D M_{2}}}$ and $d_{p}^{N I S^{\mathrm{HH}-\mathrm{D} M_{L_{2}}}}$ :

$$
\begin{gathered}
d_{p}^{P I S^{H H}-D M_{L_{2}}}=\left(\sum_{q=1}^{k_{1}} W_{1 q}\left(\frac{F_{1 q}^{*}{ }^{H H-D M_{L_{1}}}-F_{1 q}^{H H-D M_{L_{1}}}(X)}{F_{1 q}^{* H H-D M_{L_{1}}}-F_{1 q}^{-}{ }^{H H-D M_{L_{1}}}}\right)^{p}+\right. \\
\left.\sum_{q=1}^{k_{2}} W_{2 q}\left(\frac{F_{2 q}^{*}{ }^{H H-D M_{L_{2}}}-F_{2 q}^{H H-D M_{L_{2}}}(X)}{{F_{2 q}^{*}}^{H H-D M_{L_{2}}}-F_{2 q}^{-}{ }^{H H-D M_{L_{2}}}}\right)^{p}\right)^{1 / p}
\end{gathered}
$$


and

$$
\begin{gathered}
d_{p}^{N I S^{H H-D M_{L_{2}}}}=\left(\sum_{q=1}^{k_{1}} w_{1 q}\left(\frac{F_{1 q}^{H H-D M_{L_{1}}}(X)-F_{1 q}^{-}{ }^{H H-D M_{L_{1}}}}{F_{1 q}^{* H-D M_{L_{1}}-F_{1 q}^{-}}{ }^{H H-D M_{L_{1}}}}\right)^{p}+\right. \\
\left.\sum_{q=1}^{k_{2}} w_{2 q}\left(\frac{F_{2 q}^{H H-D M_{L_{2}}}(X)-F_{2 q}^{-}{ }^{H H-D M_{L_{2}}}}{F_{2 q}^{* H-D M_{L_{2}}-F_{2 q}^{-}}{ }^{H H-D M_{L_{2}}}}\right)^{{ }^{H}{ }^{1 / p}}\right)^{1 / p}
\end{gathered}
$$

Step 16:

Construct the following bi-objective problem with two commensurable (but conflicting) objectives, using the distance functions $d_{p}^{P I S^{\mathrm{LL}-D M_{L_{2}}}}$ and $d_{p}^{N I S^{\mathrm{LL}-\mathrm{D} M_{L_{2}}}}$ :

Minimize $d_{p}^{P I S^{\mathrm{LL}-D M_{L_{2}}}}(X)$

Maximize $d_{p}^{N I S^{\mathrm{LL}-\mathrm{D} M_{L_{2}}}}(X)$

subject to

$X \in \mathbb{M}$

where

$p=1,2, \ldots \ldots, \infty$.

Construct the following bi-objective problem with two commensurable (but conflicting) objectives using the distance

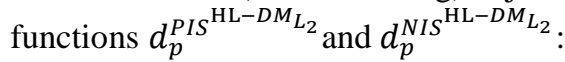

Minimize $d_{p}^{P I S^{\mathrm{HL}-D M_{L_{2}}}}(X)$

Maximize $d_{p}^{N I S^{\mathrm{HL}-D M_{L_{2}}}}(X)$

subject to

$X \in \mathbb{M}$

where

$p=1,2, \ldots, \infty$.

Construct the following bi-objective problem with two commensurable (but conflicting) objectives using the distance functions $d_{p}^{\text {PIS }}{ }^{\mathrm{LH}-D M_{L_{2}}}$ and $d_{p}^{N I S^{\mathrm{LH}-D M_{L_{2}}}}$ :

Minimize $d_{p}^{P I S^{\mathrm{LH}-D M_{L_{2}}}}(X)$

Maximize $d_{p}^{N I S^{\mathrm{LH}-D M_{L_{2}}}}(X)$

subject to

$X \in \mathbb{M}$

where

$p=1,2, \ldots, \infty$.

Construct the following bi-objective problem with two commensurable (but conflicting) objectives using the distance functions $d_{p}^{P I S^{\mathrm{HH}-D M_{L_{2}}}}$ and $d_{p}^{N I S^{\mathrm{HH}-D M_{L_{2}}}}$ :
Minimized ${ }_{p}^{\text {PIS }}{ }^{\mathrm{HH}-D M_{L_{2}}}(X)$

Maximize $d_{p}^{N I S^{\mathrm{HH}-D M_{L_{2}}}}(X)$

subject to

$X \in \mathbb{M}$

where

$p=1,2, \ldots \ldots, \infty$

\section{$\underline{\text { Step 17: }}$}

Construct PIS Payoff table for problem (39) and obtain:

$$
\begin{aligned}
& d_{p}^{-{ }^{\mathrm{LL}-D M_{L_{2}}}}=\left(\left(d_{p}^{P I S^{\mathrm{LL}-D M_{L_{2}}}}\right)^{-},\left(d_{p}^{N I S^{\mathrm{LL}-D M_{L_{2}}}}\right)^{-}\right), \\
& d_{p}^{* \mathrm{LL}-D M_{L_{2}}}=\left(\left(d_{p}^{P I S^{\mathrm{LL}-D M_{L_{2}}}}\right)^{*},\left(d_{p}^{N I S^{\mathrm{LL}-D M_{L_{2}}}}\right)^{*}\right) .
\end{aligned}
$$

where

$$
\begin{aligned}
& \left(d_{p}^{P I S^{\mathrm{LL}-D M_{L_{2}}}}\right)^{*}= \\
& \underset{X \in \mathbb{M}}{\operatorname{Minimize}} d_{p}^{P I S^{\mathrm{LL}-D M_{L_{2}}}}(X) \text { and the solution is } X^{P I S^{L L-D M_{L_{2}}}} \text {, } \\
& \left(d_{p}^{N I S^{L L-D M_{L_{2}}}}\right)^{*}=
\end{aligned}
$$

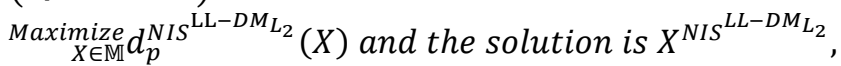

$$
\begin{aligned}
& \left(d_{p}^{P I S^{L L-D M_{L_{2}}}}\right)^{-}=d_{p}^{P I S^{\mathrm{LL}-D M_{L_{2}}}}\left(X^{N I S^{L L-D M_{L_{2}}}}\right) \text { and } \\
& \left(d_{p}^{N I S^{\mathrm{LL}-D M_{L_{2}}}}\right)^{-}=d_{p}^{N I S^{\mathrm{LL}-D M_{L_{2}}}}\left(X^{P I S^{L L-D M_{L_{2}}}}\right) .
\end{aligned}
$$

Construct PIS Payoff table for problem (40) and obtain:

$$
\begin{gathered}
d_{p}^{-{ }^{\mathrm{HL}-D M_{L_{2}}}}=\left(\left(d_{p}^{P I S^{\mathrm{HL}-D M_{L_{2}}}}\right)^{-},\left(d_{p}^{N I S^{\mathrm{HL}-D M_{L_{2}}}}\right)^{-}\right), \\
d_{p}^{*{ }^{\mathrm{HL}-D M_{L_{2}}}}=\left(\left(d_{p}^{P I S^{\mathrm{HL}-D M_{L_{2}}}}\right)^{*},\left(d_{p}^{N I S^{\mathrm{HL}-D M_{L_{2}}}}\right)^{*}\right) .
\end{gathered}
$$

Construct PIS Payoff table for problem (41) and obtain:

$$
\begin{aligned}
& d_{p}^{-{ }^{\mathrm{LH}-D M_{L_{2}}}}=\left(\left(d_{p}^{P I S^{\mathrm{LH}-D M_{L_{2}}}}\right)^{-},\left(d_{p}^{N I S^{\mathrm{LH}-D M_{L_{2}}}}\right)^{-}\right), \\
& d_{p}^{*}{ }^{\mathrm{LH}-D M_{L_{2}}}=\left(\left(d_{p}^{P I S^{\mathrm{LH}-D M_{L_{2}}}}\right)^{*},\left(d_{p}^{N I S^{\mathrm{LH}-D M_{L_{2}}}}\right)^{*}\right) .
\end{aligned}
$$

Construct PIS Payoff table for problem (42) and obtain:

$$
\begin{aligned}
& d_{p}^{-\mathrm{HH}-D M_{L_{2}}}=\left(\left(d_{p}^{P I S^{\mathrm{HH}-D M_{L_{2}}}}\right)^{-},\left(d_{p}^{N I S^{\mathrm{HH}-D M_{L_{2}}}}\right)^{-}\right), \\
& d_{p}^{*}{ }^{\mathrm{HH}-D M_{L_{2}}}=\left(\left(d_{p}^{P I S^{\mathrm{HH}-D M_{L_{2}}}}\right)^{*},\left(d_{p}^{N I S^{\mathrm{HH}-D M_{L_{2}}}}\right)^{*}\right) .
\end{aligned}
$$

\section{Step 18:}

Construct the following satisfactory level model, [11-13], (for finite value of $\mathrm{p}$ ) for problem (39): 
Maximize $\delta^{\mathrm{LL}-D M_{L_{2}}}$

subject to

$\left(\frac{d_{p}^{P I S^{\mathrm{LL}-D M_{L_{2}}}}(X)-\left(d_{p}^{P I S^{\mathrm{LL}-D M_{L_{2}}}}\right)^{*}}{\left(d_{p}^{P I S^{\mathrm{LL}-D M_{L_{2}}}}\right)^{-}-\left(d_{p}^{P I S^{\mathrm{LL}-D M_{L_{2}}}}\right)^{*}}\right) \geq \delta^{\mathrm{LL}-D M_{L_{2}}}$

$\left(\frac{\left(d_{p}^{N I S^{\mathrm{LL}-D M_{L_{2}}}}\right)^{*}-d_{p}^{N I S^{\mathrm{LL}-D M_{L_{2}}}(X)}}{\left(d_{p}^{N I S^{\mathrm{LL}-D M_{L_{2}}}}\right)^{*}-\left(d_{p}^{N I S^{\mathrm{LL}-D M_{L_{2}}}}\right)^{-}}\right) \geq \delta^{\mathrm{LL}-D M_{L_{2}}}$,

$\frac{x_{1 i^{-}}\left(x_{1 i}^{\left.* L-\mathrm{DM}_{L_{1}}-\tau_{i}^{L L-\mathrm{DM}_{L_{1}}-L}\right)}\right.}{\tau_{i}^{L L-\mathrm{DM}_{L_{1}}-L}} \geq \delta^{\mathrm{LL}-\mathrm{DM}_{L_{2}}}, i=1,2, \ldots, n_{1}$,

$\frac{\left(X_{1 i}^{\left.* L-\mathrm{DM}_{L_{1}}+\tau_{i}^{L L-\mathrm{DM}_{L_{1}}-R}\right)-x_{1 i}}\right.}{\tau_{i}^{L L-\mathrm{DM}_{L_{1}}-R}} \geq \delta^{\mathrm{LL}-\mathrm{DM}_{L_{2}}}, i=1,2, \ldots, n_{1}$

$X \in \mathbb{M}, \delta^{\mathrm{LL}-D M_{L_{2}}} \in[0,1]$

Construct the following satisfactory level model (for finite value of $\mathrm{p}$ ) for problem (40):

Maximize $\delta^{\mathrm{HL}-D M_{L_{2}}}$

subject to

$\left(\frac{d_{p}^{P I S^{\mathrm{HL}-D M_{L_{2}}}}(X)-\left(d_{p}^{P I S^{\mathrm{HL}-D M_{L_{2}}}}\right)^{*}}{\left(d_{p}^{P I S^{\mathrm{HL}-D M_{L_{2}}}}\right)^{-}-\left(d_{p}^{P I S^{\mathrm{HL}-D M_{L_{2}}}}\right)^{*}}\right) \geq \delta^{\mathrm{HL}-D M_{L_{2}}}$,

$\left(\frac{\left(d_{p}^{N I S^{\mathrm{HL}-D M_{L_{2}}}}\right)^{*}-d_{p}^{N I S^{\mathrm{HL}-D M_{L_{2}}}(X)}}{\left(d_{p}^{N I S^{\mathrm{HL}-D M_{L_{2}}}}\right)^{*}-\left(d_{p}^{N I S^{\mathrm{HL}-D M_{L_{2}}}}\right)}\right) \geq \delta^{\mathrm{HL}-D M_{L_{2}}}$,

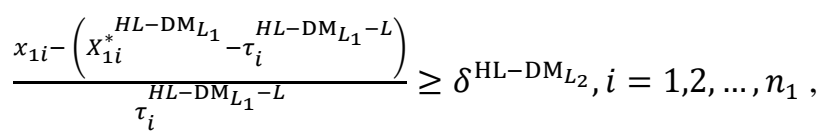

$\frac{\left(X_{1 i}^{*}{ }^{H L-\mathrm{DM}_{L_{1}}}+\tau_{i}^{H L-\mathrm{DM}_{L_{1}}-R}\right)-x_{1 i}}{\tau_{i}^{H L-\mathrm{DM}_{L_{1}}-R}} \geq \delta^{\mathrm{HL}-\mathrm{DM}_{L_{2}}}, i=1,2, \ldots, n_{1}$

$X \in \mathbb{M}, \delta^{\mathrm{HL}-D M_{L_{2}}} \in[0,1]$.

Construct the following satisfactory level model (for finite value of p) for problem (41):

Maximize $\delta^{\mathrm{LH}-D M_{L_{2}}}$

subject to

$\left(\frac{d_{p}^{P I S^{\mathrm{LH}-D M_{L_{2}}}}(X)-\left(d_{p}^{P I S^{\mathrm{LH}-D M_{L_{2}}}}\right)^{*}}{\left(d_{p}^{P I S^{\mathrm{LH}-D M_{L_{2}}}}\right)^{-}-\left(d_{p}^{P I S^{\mathrm{LH}-D M_{L_{2}}}}\right)^{*}}\right) \geq \delta^{\mathrm{LH}-D M_{L_{2}}}$,

$\left(\frac{\left(d_{p}^{N I S^{\mathrm{LH}-D M_{L_{2}}}}\right)^{*}-d_{p}^{N I S^{\mathrm{LH}-D M_{L_{2}}}(X)}}{\left(d_{p}^{N I S^{\mathrm{LH}-D M_{L_{2}}}}\right)^{*}-\left(d_{p}^{N I S^{\mathrm{LH}-D M_{L_{2}}}}\right)}\right) \geq \delta^{\mathrm{LH}-D M_{L_{2}}}$, $\frac{x_{1 i}-\left(X_{1 i}^{*}{ }^{L H-\mathrm{DM}_{L_{1}}}-\tau_{i}^{L H-\mathrm{DM}_{L_{1}}-L}\right)}{\tau_{i}^{L H-\mathrm{DM}_{L_{1}}-L}} \geq \delta^{\mathrm{LH}-\mathrm{DM}_{L_{2}}}, i=1,2, \ldots, n_{1}$,

$\frac{\left(x_{1 i}^{*}{ }^{L H-\mathrm{DM}_{L_{1}}}+\tau_{i}^{L \mathrm{LH}-\mathrm{DM}_{L_{1}}-R}\right)-x_{1 i}}{\tau_{i}^{L H-\mathrm{DM}_{L_{1}}-R}} \geq \delta^{\mathrm{LH}-\mathrm{DM}_{L_{2}}}, i=1,2, \ldots, n_{1}$,

$X \in \mathbb{M}, \delta^{\mathrm{LH}-D M_{L_{2}}} \in[0,1]$.

Construct the following satisfactory level model (for finite value of $p$ ) for problem (42):

Maximize $\delta^{\mathrm{HH}-D M_{L_{2}}}$

subject to

$\left(\frac{d_{p}^{P I S^{\mathrm{HH}-D M_{L_{2}}}}(X)-\left(d_{p}^{P I S^{\mathrm{HH}-D M_{L_{2}}}}\right)^{*}}{\left(d_{p}^{P I S^{\mathrm{HH}-D M_{L_{2}}}}\right)^{-}-\left(d_{p}^{P I S^{\mathrm{HH}-D M_{L_{2}}}}\right)^{*}}\right) \geq \delta^{\mathrm{HH}-D M_{L_{2}}}$,

$\left(\frac{\left(d_{p}^{N I S^{\mathrm{HH}-D M_{L_{2}}}}\right)^{*}-d_{p}^{N I S^{\mathrm{HH}-D M_{L_{2}}}(X)}}{\left(d_{p}^{N I S^{\mathrm{HH}-D M_{L_{1}}}}\right)^{*}-\left(d_{p}^{N I S^{\mathrm{HH}-D M_{L_{1}}}}\right)^{-}}\right) \geq \delta^{\mathrm{HH}-D M_{L_{2}}}$,

$\frac{x_{1 i}-\left(x_{1 i}^{*}{ }^{H H-\mathrm{DM}_{L_{1}}}-\tau_{i}^{H H-\mathrm{DM}_{L_{1}}-L}\right)}{\tau_{i}^{H H-\mathrm{DM}_{L_{1}}-L}} \geq \delta^{\mathrm{HH}-\mathrm{DM}_{L_{2}}}, i=1,2, \ldots, n_{1}$,

$\frac{\left(X_{1 i}^{* H H-\mathrm{DM}_{L_{1}}}+\tau_{i}^{H \mathrm{H}-\mathrm{DM}_{L_{1}}-R}\right)-x_{1 i}}{\tau_{i}^{H H-\mathrm{DM}_{L_{1}}-R}} \geq \delta^{\mathrm{HH}-\mathrm{DM}_{L_{2}}}, i=1,2, \ldots, n_{1}$,

$X \in \mathbb{M}, \delta^{\mathrm{HH}-D M_{L_{2}}} \in[0,1]$.

\section{Step 19:}

Solve problems (43-468) to obtain the satisfactory levels $\delta^{*^{\mathrm{LL}-D M_{L_{2}}}}, \delta^{*^{\mathrm{HL}-D M_{L_{2}}}}, \delta^{{ }{ }^{\mathrm{LH}-D M_{L_{2}}}}$ and $\delta^{{ }^{\mathrm{HH}-D M_{L_{2}}}}$ for the compromise solutions $X^{*^{\mathrm{LL}-D M_{L_{2}}}}, X^{*^{\mathrm{HL}-D M_{L_{2}}}}, X^{*^{\mathrm{LH}-D M_{L_{2}}}}$ and $X^{*}{ }^{\mathrm{HH}-D M_{L_{2}}}$. If the $\mathrm{DM}_{L_{2}}$ is satisfied with the solutions, then go to step (20). Otherwise, go to step (14).

\section{$\underline{\text { Step 20: }}$}

Ask the $\mathrm{DM}_{L_{2}}$ to select the maximum acceptable negative and positive tolerance (relaxation) values:

$$
\begin{aligned}
& \tau_{i}^{\mathrm{LL}-D M_{L_{2}}-L}, \tau_{i}^{\mathrm{LL}-D M_{L_{2}}-R}, \tau_{i}^{\mathrm{HL}-D M_{L_{2}}-L}, \tau_{i}^{\mathrm{HL}-D M_{L_{2}}-R}, \\
& \tau_{i}^{\mathrm{LH}-D M_{L_{2}}-L}, \tau_{i}^{\mathrm{LH}-D M_{L_{2}}-R}, \tau_{i}^{\mathrm{HH}-D M_{L_{2}}-L} \text { and } \tau_{i}^{\mathrm{HH}-D M_{L_{2}}-R}, i= \\
& \quad 1,2, \ldots, n_{2}
\end{aligned}
$$

on the decision vectors:

$$
\begin{aligned}
& X_{2}^{*{ }^{\mathrm{LL}-D M_{L_{2}}}}=\left(x_{21}^{*{ }^{\mathrm{LL}-D M_{L_{2}}}}, x_{22}^{*}{ }^{\mathrm{LL}-D M_{L_{2}}}, \ldots, x_{2 n_{2}}^{* \mathrm{LL}-D M_{L_{2}}}\right), \\
& X_{2}^{*{ }^{H L}-D M_{L_{2}}}=\left(x_{21}^{* \mathrm{HL}-D M_{L_{2}}}, x_{22}^{* \mathrm{HL}-D M_{L_{2}}}, \ldots, x_{2 n_{2}}^{* \mathrm{HL}-D M_{L_{2}}}\right), \\
& X_{2}^{*}{ }^{\mathrm{LH}-D M_{L_{2}}}=\left(x_{21}^{*}{ }^{\mathrm{LH}-D M_{L_{2}}}, x_{22}^{*}{ }^{\mathrm{LH}-D M_{L_{2}}}, \ldots, x_{2 n_{2}}^{\mathrm{LH}^{\mathrm{LH}}-D M_{L_{2}}}\right),
\end{aligned}
$$


$X_{2}^{*}{ }^{\mathrm{HH}-D M_{L_{2}}}=\left(x_{21}^{* \mathrm{HH}-D M_{L_{2}}}, x_{22}^{* \mathrm{HH}-D M_{L_{2}}}, \ldots, x_{2 n_{2}}^{*^{\mathrm{HH}-D M_{L_{2}}}}\right)$.

Set $\mathrm{i}=\mathrm{i}+1$, if $\mathrm{i} \leq \mathrm{h}$ goes to the next phase. Otherwise, stop.

\section{ILLUSTRATIVE NUMERICAL EXAMPLE}

Consider the following linear multi-level multi-objective decision making (LMLMODM) problem with rough parameters in the objective functions

\section{First Level:}

Maximize $\mathrm{f}_{11}(\mathrm{X})=2([2,3],[1,5]) \mathrm{x}_{1}+([3,5],[2,7]) \mathrm{x}_{2}+$ $([2,3],[1,4]) x_{3}$

Maximize $f_{12}(X)=([6,7],[5,9]) x_{1}-x_{2}+$ $([1,3],[1,6]) x_{3}+([1,3],[0,5])$

\section{Second Level:}

Maximize $f_{21}(X)=2 x_{1}+([5,6],[3,8]) x_{2}-$ $2([0,3],[0,6]) x_{3}+([5,6],[3,7])$

Maximize $f_{22}(X)=x_{1}-([3,4],[2,6]) x_{2}+$ $([1,3],[1,7]) x_{3}+([3,4],[2,6])$

Third Level:

Maximize $f_{31}(X)=([2,5],[1,8]) x_{1}+2 x_{2}+x_{3}+$ $([4,5],[3,6])$

Maximize $f_{32}(X)=5 x_{1}+2([1,2],[1,4]) x_{2}-x_{3}+$ $([6,7],[5,8])$

Subject to:

$\mathrm{X} \in \mathbb{M}=\left\{3 \mathrm{x}_{1}+5 \mathrm{x}_{2}+\mathrm{x}_{3} \leq 35,2 \mathrm{x}_{1}-\mathrm{x}_{2}+12 \mathrm{x}_{3} \leq\right.$ $\left.20,5 x_{2}+6 x_{3} \leq 16, x_{1} \geq 1, x_{1}, x_{2}, x_{3} \geq 0\right\}$

Solution:

Phase (1):

$$
\underline{\mathbf{P}^{\mathrm{LL}}:}
$$

First Level:

MaximizeF $_{11}^{\mathrm{LL}}=4 x_{1}+3 x_{2}+x_{3}+2$

Maximize $\mathrm{F}_{12}^{\mathrm{LL}}=6 x_{1}-x_{2}+x_{3}+1$

$\underline{\text { Second Level: }}$

$\operatorname{MaximizeF}_{21}^{\mathrm{LL}}=2 x_{1}+5 x_{2}+5$

Maximize $\mathrm{F}_{22}^{\mathrm{LL}}=x_{1}-3 x_{2}+x_{3}+3$

Third Level:

$\operatorname{MaximizeF}_{31}^{\mathrm{LL}}=2 x_{1}-2 x_{2}+x_{3}+4$

Maximize $\mathrm{F}_{32}^{\mathrm{LL}}=5 x_{1}+2 x_{2}-x_{3}+6$
$\mathrm{X} \in \mathbb{M}$

$$
\underline{\mathbf{P}^{\mathrm{HL}}:}
$$

$\underline{\text { First Level: }}$

$\operatorname{MaximizeF}_{11}^{\mathrm{HL}}=6 x_{1}+5 x_{2}+x_{3}+3$

Maximize $\mathrm{F}_{12}^{\mathrm{HL}}=7 x_{1}-x_{2}+3 x_{3}+3$

$\underline{\text { Second Level: }}$

$\operatorname{MaximizeF}_{21}^{\mathrm{HL}}=2 x_{1}+6 x_{2}++6 x_{3}+6$

Maximize $\mathrm{F}_{22}^{\mathrm{HL}}=x_{1}-4 x_{2}+3 x_{3}+4$

Third Level:

$\operatorname{MaximizeF}_{31}^{\mathrm{HL}}=5 x_{1}-2 x_{2}+x_{3}+5$

Maximize $\mathrm{F}_{32}^{\mathrm{HL}}=5 x_{1}+4 x_{2}-x_{3}+7$

$\underline{\text { Subject to }}$

$\mathrm{X} \in \mathbb{M}$

$\underline{\mathbf{P}^{\mathrm{LH}}:}$

First Level:

$\operatorname{MaximizeF}_{11}^{\mathrm{LH}}=2 x_{1}+2 x_{2}+x_{3}+1$

Maximize $\mathrm{F}_{12}^{\mathrm{LH}}=5 x_{1}-x_{2}+x_{3}$

$\underline{\text { Second Level: }}$

$\operatorname{MaximizeF}_{21}^{\mathrm{LH}}=2 x_{1}+3 x_{2}+3$

Maximize $\mathrm{F}_{22}^{\mathrm{LH}}=x_{1}-2 x_{2}+x_{3}+2$

Third Level:

$\operatorname{MaximizeF}_{31}^{\mathrm{HL}}=x_{1}-2 x_{2}+x_{3}+3$

Maximize $\mathrm{F}_{32}^{\mathrm{HL}}=5 x_{1}+2 x_{2}-x_{3}+5$

$\underline{\text { Subject to }}$

$X \in \mathbb{M}$

$\underline{\mathbf{P}^{\mathrm{HH}}:}$

First Level:

$\operatorname{MaximizeF}_{11}^{\mathrm{HH}}=10 x_{1}+7 x_{2}+x_{3}+4$

Maximize $\mathrm{F}_{12}^{\mathrm{HH}}=9 x_{1}-x_{2}+6 x_{3}+5$

Second Level:

$\operatorname{MaximizeF}_{21}^{\mathrm{HH}}=2 x_{1}+8 x_{2}-12 x_{3}+7$

Maximize $\mathrm{F}_{22}^{\mathrm{HH}}=x_{1}-6 x_{2}+7 x_{3}+6$ 
Third Level:

$\operatorname{MaximizeF}_{31}^{\mathrm{HH}}=8 x_{1}-2 x_{2}+x_{3}+6$

Maximize $\mathrm{F}_{32}^{\mathrm{HH}}=5 x_{1}+8 x_{2}-x_{3}+8$

Subject to

$\mathrm{X} \in \mathbb{M}$

$$
\underline{\mathbf{P}^{\mathrm{LL}}:}
$$

(First Level):

Obtain PIS and NIS payoff tables for problem $\mathrm{P}^{\mathrm{LL}}$ :

Table 1. PIS payoff table for problem $\mathrm{P}^{\mathrm{LL}}$

\begin{tabular}{lccccc}
\hline & $\boldsymbol{F}_{11}^{L L}(\boldsymbol{X})$ & $\boldsymbol{F}_{\mathbf{1 2}}^{\boldsymbol{L L}}(\boldsymbol{X})$ & $\boldsymbol{x}_{\mathbf{1}}$ & $\boldsymbol{x}_{\mathbf{2}}$ & $\boldsymbol{x}_{\mathbf{3}}$ \\
\hline $\operatorname{Max.}_{11}^{L L}(X)$ & $45.85^{*}$ & 62.54 & 10.38 & 0.77 & 0 \\
$\operatorname{Max} F_{12}^{L L}(X)$ & 45.85 & $62.54^{*}$ & 10.38 & 0.77 & 0 \\
\hline
\end{tabular}

PIS: $\mathrm{f}^{* \mathrm{LL}}=(45.84615,62.53846)$

Table 2. NIS payoff table for problem $\mathrm{P}^{\mathrm{LL}}$

\begin{tabular}{lcllll}
\hline & $\boldsymbol{F}_{1}^{L L}(\boldsymbol{X})$ & $\boldsymbol{F}_{\mathbf{2}}^{\boldsymbol{L L}}(\boldsymbol{X})$ & $\boldsymbol{x}_{\mathbf{1}}$ & $\boldsymbol{x}_{\mathbf{2}}$ & $\boldsymbol{x}_{\mathbf{3}}$ \\
\hline $\operatorname{Min} . F_{11}^{L L}(X)$ & $2^{-}$ & 1 & 0 & 0 & 0 \\
$\operatorname{Min} . F_{12}^{L L}(X)$ & 11.6 & $-2.2^{-}$ & 0 & 3.2 & 0 \\
\hline
\end{tabular}

NIS: $f^{-L L}=(2,-2.2)$

Next, construct equation and obtain the following equations:

$$
\begin{gathered}
d_{p}^{P I S^{L L-D M_{L_{1}}}}=\left[w_{1}^{p}\left(\frac{45.84615-F_{11}^{L L}(X)}{45.84615-2}\right)^{p}+\right. \\
\left.w_{2}^{p}\left(\frac{62.53846-F_{12}^{L L}(X)}{62.53846-(-2.2)}\right)^{p}\right]^{1 / p}
\end{gathered}
$$

$d_{p}^{N I S^{L L-D M_{L_{1}}}}=\left[w_{1}^{p}\left(\frac{F_{11}^{L L}(X)-(2)}{45.84615-2}\right)^{p}+w_{2}^{p}\left(\frac{F_{12}^{L L}(X)-(-2.2)}{62.53846-(-2.2)}\right)^{p}\right]^{1 / p}$

Thus, problem (11) is obtained. In order to get numerical solutions, assume that $w_{1}^{p}=w_{2}^{p}=0.5$ and $\mathrm{p}=2$

Table 3. PIS payoff table of problem (11) when $\mathrm{p}=2$

\begin{tabular}{lccccccc}
\hline & $\mathbf{d}_{\mathbf{2}}^{\text {PIS }}$ & $\mathbf{d}_{\mathbf{2}}^{\text {NIS }}$ & $\mathbf{F}_{11}^{\mathrm{LL}}(\mathbf{X})$ & $\mathbf{F}_{\mathbf{1 2}}^{\mathrm{LL}}(\mathbf{X})$ & $\mathbf{x}_{\mathbf{1}}$ & $\mathbf{x}_{\mathbf{2}}$ & $\mathbf{x}_{\mathbf{3}}$ \\
\hline Min. $\mathrm{d}_{2}^{\mathrm{PI} S^{\mathrm{LL}}}$ & $22.40^{*}$ & $0.71^{-}$ & 45.9 & 62.5 & $\begin{array}{c}10 . \\
4\end{array}$ & $\begin{array}{c}0 . \\
8\end{array}$ & 0 \\
Max. $_{2}^{\text {NIS }}$ & 0 & $0.56^{*}$ & 45.85 & 62.5 & $\begin{array}{c}10 . \\
4\end{array}$ & $\begin{array}{c}0 . \\
8\end{array}$ & 0 \\
\hline
\end{tabular}

$d_{2}^{*^{L L}}=(22.40027,0.5590170), d_{2}^{-L L}=(0,0.7071067812)$.

Now, it is easy to compute step (10):

Maximize $\delta^{L L-D M_{L_{1}}}$

Subject to

$$
\begin{gathered}
3 x_{1}+5 x_{2}+x_{3} \leq 35,2 x_{1}-x_{2}+12 x_{3} \leq 20,5 x_{2}+6 x_{3} \leq \\
16, x_{1} \geq 1, x_{1}, x_{2}, x_{3} \geq 0
\end{gathered}
$$

$$
\begin{aligned}
& \left(\frac{d_{1}^{P I S}{ }^{L L}}{0.5590170-22.40027}\right) \geq \delta^{L L-D M_{L_{1}}}, \\
& \left(\frac{{\text { zero- }-d_{1}^{N I S}}^{L L}(X)}{\text { zero- } 0.7071067812}\right) \geq \delta^{L L-D M_{L_{1}}}, \delta^{L L-D M_{L_{1}}} \in[0,1] .
\end{aligned}
$$

\begin{tabular}{|c|c|c|c|c|c|}
\hline & $F_{21}^{L L}(X)$ & $F_{22}^{L L}(X)$ & $x_{1}$ & $x_{2}$ & $x_{3}$ \\
\hline $\operatorname{Max}_{21}^{L L}(X)$ & $33.6667^{*}$ & $\begin{array}{c}- \\
0.2667\end{array}$ & 6.3333 & 3.2 & 0 \\
\hline $\operatorname{Max}_{22}^{L L}(X)$ & 25 & $13^{*}$ & 10 & 0 & 0 \\
\hline
\end{tabular}

The maximum "satisfactory level" $\left(\delta^{L L-D M_{L_{1}}}=0.9447279 \mathrm{E}\right.$ $07)$ is achieved for the solution $x_{1}^{* L L}=10.38462$, $x_{2}^{* L L}=0.7692308, x_{3}^{* L L}=$ zero.

\section{(Second Level)}

Obtain PIS and NIS payoff tables for problem $P^{L L}$ :

Table 4. PIS payoff table for problem $P^{L L}$

PIS: $f^{* L L}=(33.6667,13)$

Table 5. NIS payoff table for problem $P^{\mathbf{L L}}$

\begin{tabular}{lccccc}
\hline & $\boldsymbol{F}_{\mathbf{2 1}}^{\mathbf{L L}}(\boldsymbol{X})$ & $\boldsymbol{F}_{\mathbf{2}}^{\mathbf{L L}}(\boldsymbol{X})$ & $\boldsymbol{x}_{\mathbf{1}}$ & $\boldsymbol{x}_{\mathbf{2}}$ & $\boldsymbol{x}_{\mathbf{3}}$ \\
\hline $\operatorname{Min} F_{21}^{L L}(X)$ & $5^{-}$ & 0 & 0 & 0 & 0 \\
$\operatorname{Min} . F_{22}^{L L}(X)$ & 21 & $-6.6^{-}$ & 0 & 3.2 & 0 \\
\hline
\end{tabular}

NIS: $f^{-L L}=(5,-6.6)$

Next, construct equation and obtain the following equations:

\begin{tabular}{|c|c|c|c|c|c|c|c|}
\hline & $\mathrm{d}_{2}^{\mathrm{PI} \mathrm{S}^{\mathrm{LL}}}$ & $\mathrm{d}_{2}^{\mathrm{NIS}}{ }^{\mathrm{LL}}$ & $F_{21}^{L L}(X)$ & $F_{22}^{L L}(X)$ & $\mathbf{x}_{1}$ & $\mathbf{x}_{2}$ & $\mathbf{x}_{3}$ \\
\hline Min. $d_{2}^{P I S L L}$ & $11.2^{*}$ & $0.47^{-}$ & 29.62 & 11.1 & $\begin{array}{c}10 . \\
4\end{array}$ & $\begin{array}{c}0.7 \\
7\end{array}$ & 0 \\
\hline $\operatorname{Max} . d_{2}^{N I S^{L L}}$ & 0.04 & $0.17^{*}$ & 29.62 & 11.1 & $\begin{array}{c}10 . \\
4\end{array}$ & $\begin{array}{c}0.7 \\
7\end{array}$ & 0 \\
\hline
\end{tabular}

$$
\begin{aligned}
& d_{p}^{P I S^{L L-D M_{L_{2}}}}=\left[w_{1}^{p}\left(\frac{45.84615-F_{11}^{L L}(X)}{45.84615-2}\right)^{p}+\right. \\
& w_{2}^{p}\left(\frac{62.53846-F_{12}^{L L}(X)}{62.53846-(-2.2)}\right)^{p}+w_{3}^{p}\left(\frac{33.6667-F_{21}^{L L}(X)}{33.6667-5}\right)^{p}+ \\
& \left.w_{4}^{p}\left(\frac{13-F_{22}^{L L}(X)}{13-(-6.6)}\right)^{p}\right]^{1 / p}
\end{aligned}
$$

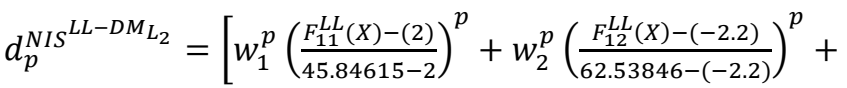

$$
\begin{aligned}
& \left.w_{3}^{p}\left(\frac{F_{21}^{L L}(X)-(5)}{33.6667-5}\right)^{p}+w_{4}^{p}\left(\frac{F_{22}^{L L}(X)-(-6.6)}{13-(-6.6)}\right)^{p}\right]^{1 / p}
\end{aligned}
$$

Thus, problem (11) is obtained. In order to get numerical solutions, assume that $w_{1}^{p}=w_{2}^{p}=w_{3}^{p}=w_{4}^{p}=0.25$ and $\mathrm{p}=2$,

Table 6. PIS payoff table of problem (11) when $\mathrm{p}=2$

$d_{2}^{*^{L L}}=(11.20014,0.1688567), d_{2}^{-L L}=(0.04301006758$, 0.4710896811).

Now, it is easy to compute step (10):

Maximize $\delta^{L L-D M_{L_{2}}}$ 
Subject to

$3 x_{1}+5 x_{2}+x_{3} \leq 35,2 x_{1}-x_{2}+12 x_{3} \leq 20,5 x_{2}+6 x_{3} \leq$ $16, x_{1} \geq 1, x_{1}, x_{2}, x_{3} \geq 0$

$\left(\frac{d_{1}^{P I S^{L L}}(X)-11.20014}{0.1688567-11.20014}\right) \geq \delta^{L L-D M_{L_{2}}}$

$\left(\frac{0.04301006758-d_{1}^{N I S^{L L}}(X)}{0.04301006758-0.4710896811}\right) \geq \delta^{L L-D M_{L_{2}}}$,

$\delta^{L L-D M_{L_{2}}} \in[0,1]$

$\left(\frac{(10.38462+0.001)-x_{1}}{0.001}\right) \geq \delta^{L L-D M_{L_{2}}}$

$\left(\frac{x_{1}-(10.38462-0.001)}{0.001}\right) \geq \delta^{L L-D M_{L_{2}}}$

The maximum "satisfactory level" $\left(\delta^{L L-D M_{L_{2}}}=0.7886892 \mathrm{E}\right.$ $07)$ is achieved for the solution $x_{1}^{* L L}=10.38462$, $x_{2}^{* L L}=0.7692308, x_{3}^{* L L}=$ zero

(Third Level):

Obtain PIS and NIS payoff tables for problem $P^{L L}$ :

Table 7. PIS payoff table for problem $\mathrm{P}^{\mathrm{LL}}$

\begin{tabular}{lccccc}
\hline & $\boldsymbol{F}_{31}^{\boldsymbol{L L}}(\boldsymbol{X})$ & $\boldsymbol{F}_{\mathbf{3 2}}^{\boldsymbol{L L}}(\boldsymbol{X})$ & $\boldsymbol{x}_{\mathbf{1}}$ & $\boldsymbol{x}_{\mathbf{2}}$ & $\boldsymbol{x}_{\mathbf{3}}$ \\
\hline $\operatorname{Max} . F_{31}^{L L}(X)$ & $24^{*}$ & 56 & 10 & 0 & 0 \\
$\operatorname{Max} . F_{32}^{L L}(X)$ & 23.23 & $59.46^{*}$ & 10.38 & 0.77 & 0 \\
\hline
\end{tabular}

PIS: $f^{* L}=(24,59.46154)$

Table 8. NIS payoff table for problem PLL Table 8. NIS payoff table for problem $P^{L L}$

\begin{tabular}{lccccc}
\hline & $\boldsymbol{F}_{\mathbf{3 1}}^{\boldsymbol{L L}}(\boldsymbol{X})$ & $\boldsymbol{F}_{\mathbf{3 2}}^{\boldsymbol{L L}}(\boldsymbol{X})$ & $\boldsymbol{x}_{\mathbf{1}}$ & $\boldsymbol{x}_{\mathbf{2}}$ & $\boldsymbol{x}_{\mathbf{3}}$ \\
\hline $\operatorname{Min} F_{31}^{L L}(X)$ & $-2.4^{-}$ & 12.4 & 0 & 3.2 & 0 \\
$\operatorname{Min} . F_{32}^{L L}(X)$ & 5.67 & $4.3^{-}$ & 0 & 0 & 1.67 \\
\hline
\end{tabular}

NIS: $f^{-L L}=(-2.4,4.3)$

Next, construct equation and obtain the following equations:

$$
\begin{aligned}
d_{p}^{P I S}{ }^{L L-D M_{L_{3}}}=\left[w_{1}^{p}\left(\frac{45.84615-F_{11}^{L L}(X)}{45.84615-2}\right)^{p}+\right. \\
w_{2}^{p}\left(\frac{62.53846-F_{12}^{L L}(X)}{62.53846-(-2.2)}\right)^{p}+w_{3}^{p}\left(\frac{33.6667-F_{21}^{L L}(X)}{33.6667-5}\right)^{p}+ \\
w_{4}^{p}\left(\frac{13-F_{22}^{L L}(X)}{13-(-6.6)}\right)^{p}+w_{5}^{p}\left(\frac{24-F_{31}^{L L}(X)}{24-(-2.4)}\right)^{p}+ \\
\\
\left.w_{6}^{p}\left(\frac{59.46154-F_{32}^{L L}(X)}{59.46154-(4.3)}\right)^{p}\right]^{1 / p}
\end{aligned}
$$

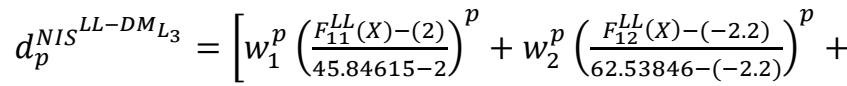

$w_{3}^{p}\left(\frac{F_{21}^{L L}(X)-(5)}{33.6667-5}\right)^{p}+w_{4}^{p}\left(\frac{F_{22}^{L L}(X)-(-6.6)}{13-(-6.6)}\right)^{p}+$

$\left.w_{5}^{p}\left(\frac{F_{31}^{L L}(X)-(-2.4)}{24-(-2.4)}\right)^{p}+w_{6}^{p}\left(\frac{F_{32}^{L L}(X)-(4.3)}{59.46154-(4.3)}\right)^{p}\right]^{1 / p}$

\begin{tabular}{|c|c|c|c|c|c|c|c|}
\hline & $d_{3}^{P I S^{L L}}$ & $d_{3}^{N I S^{L L}}$ & $F_{31}^{L L}(X)$ & $F_{32}^{L L}(X)$ & $x_{1}$ & $x_{2}$ & $x_{3}$ \\
\hline $\operatorname{Min} . d_{3}^{P I S^{L L}}$ & $7.47^{*}$ & $0.35^{-}$ & 23.23 & 59.46 & $\begin{array}{c}10 . \\
4\end{array}$ & $\begin{array}{c}0.7 \\
7\end{array}$ & 0 \\
\hline $\operatorname{Max} . d_{3}^{N I S^{L L}}$ & 0.03 & $0.10^{*}$ & 23.23 & 59.46 & $\begin{array}{c}10 . \\
4\end{array}$ & $\begin{array}{c}0.7 \\
7\end{array}$ & 0 \\
\hline
\end{tabular}

Thus, problem (11) is obtained. In order to get numerical solutions, assume that $w_{1}^{p}=w_{2}^{p}=w_{3}^{p}=w_{4}^{p}=w_{5}^{p}=w_{6}^{p}=1 / 6$ and $\mathrm{p}=2$,

Table 9. PIS payoff table of problem (11) when $\mathrm{p}=2$

$d_{3}^{* L L}=(7.466758,0.1035186), d_{3}^{-L L}=(0.02925962351$, $0.3532933399)$.

Now, it is easy to compute step (10):

Maximize $\delta^{L L-D M_{L_{3}}}$

Subject to

$3 x_{1}+5 x_{2}+x_{3} \leq 35,2 x_{1}-x_{2}+12 x_{3} \leq 20,5 x_{2}+6 x_{3} \leq$ $16, x_{1} \geq 1, x_{1}, x_{2}, x_{3} \geq 0$

$\left(\frac{d_{3}^{P I S^{L L}}(X)-7.466758}{0.1035186-7.466758}\right) \geq \delta^{L L-D M_{L_{3}}}$

$\left(\frac{0.02925962351-d_{3}^{N I S^{L L}}(X)}{0.02925962351-0.3532933399}\right) \geq \delta^{L L-D M_{L_{3}}}$

$\delta^{L L-D M_{L_{3}}} \in[0,1]$

$\left(\frac{(0+0.001)-+x_{3}}{0.001}\right) \geq \delta^{L L-D M_{L_{3}}},\left(\frac{x_{3}-(0-0.001)}{0.001}\right) \geq \delta^{L L-D M_{L_{3}}}$

The maximum "satisfactory level" $\left(\delta^{L L-D M_{L_{3}}}=0.6082756 \mathrm{E}\right.$ $07)$ is achieved for the solution $x_{1}^{* L L}=10.38462$, $x_{2}^{* L L}=0.7692308, x_{3}^{* L L}=$ zero.

(First Level):

\section{$\underline{P^{H L}:}$}

Obtain PIS and NIS payoff tables for problem $P^{H L}$ :

Table 10. PIS payoff table for problem $P^{H L}$

\begin{tabular}{lccccc}
\hline & $\boldsymbol{F}_{\mathbf{1 1}}^{\boldsymbol{H L}}(\boldsymbol{X})$ & $\boldsymbol{F}_{\mathbf{1 2}}^{\boldsymbol{H}}(\boldsymbol{X})$ & $\boldsymbol{x}_{\mathbf{1}}$ & $\boldsymbol{x}_{\mathbf{2}}$ & $\boldsymbol{x}_{\mathbf{3}}$ \\
\hline $\operatorname{Max} . F_{11}^{H L}(X)$ & $69.15^{*}$ & 74.92 & 10.38 & 0.77 & 0 \\
$\operatorname{Max} . F_{12}^{H L}(X)$ & 69.15 & $74.92^{*}$ & 10.38 & 0.77 & 0 \\
\hline
\end{tabular}

PIS: $f^{* H L}=(69.15385,74.92308)$

Table 11. NIS payoff table for problem $P^{H L}$

\begin{tabular}{lccccc}
\hline & $\boldsymbol{F}_{\mathbf{1}}^{\boldsymbol{H}}(\boldsymbol{X})$ & $\boldsymbol{F}_{\mathbf{2}}^{\boldsymbol{H}}(\boldsymbol{X})$ & $\boldsymbol{x}_{\mathbf{1}}$ & $\boldsymbol{x}_{\mathbf{2}}$ & $\boldsymbol{x}_{\mathbf{3}}$ \\
\hline $\operatorname{Min} . F_{11}^{H L}(X)$ & $3^{-}$ & 3 & 0 & 0 & 0 \\
$\operatorname{Min} . F_{12}^{H L}(X)$ & 19 & $-0.2^{-}$ & 0 & 3.2 & 0 \\
\hline
\end{tabular}

NIS: $f^{-L L}=(3,-0.2)$

Next, construct equation and obtain the following equations: 


$$
\begin{gathered}
d_{p}^{P I S^{H L-D M_{L_{1}}}}=\left[w_{1}^{p}\left(\frac{69.15385-F_{11}^{H L}(X)}{69.15385-3}\right)^{p}+\right. \\
\left.w_{2}^{p}\left(\frac{74.92308-F_{12}^{H L}(X)}{74.92308-(-0.2)}\right)^{p}\right]^{1 / p} \\
d_{p}^{N I S^{H L-D M_{L_{1}}}}=\left[w_{1}^{p}\left(\frac{F_{11}^{H L}(X)-(3)}{69.15385-3}\right)^{p}+\right. \\
\left.w_{2}^{p}\left(\frac{F_{12}^{H L}(X)-(-0.2)}{74.92308-(-0.2)}\right)^{p}\right]^{1 / p}
\end{gathered}
$$

Thus, problem (11) is obtained. In order to get numerical solutions, assume that $w_{1}^{p}=w_{2}^{p}=0.5$ and $\mathrm{p}=2$,

Table 12. PIS payoff table of problem (11) when $\mathrm{p}=2$

\begin{tabular}{lccccccc}
\hline & $\boldsymbol{d}_{\mathbf{2}}^{\text {PIS }}$ & $\boldsymbol{d}_{\mathbf{2}}^{\text {NIS }}$ & $\boldsymbol{F}_{\mathbf{1 1}}^{H \boldsymbol{L}}$ & $\boldsymbol{F}_{\mathbf{1 2}} \boldsymbol{H L}$ & $\boldsymbol{x}_{\mathbf{1}}$ & $\boldsymbol{x}_{\mathbf{2}}$ & $\boldsymbol{x}_{\mathbf{3}}$ \\
\hline Min. $d_{2}^{\text {PIS }}$ & $34.58^{*}$ & $0.71^{-}$ & 69.1 & 74.9 & 10.3 & 0.7 & 0 \\
& & & 5 & 2 & 8 & 7 & \\
Max. $d_{2}^{\text {NIS }}$ & 0 & $0.56^{*}$ & 69.1 & 74.9 & 10.3 & 0.7 & 0 \\
& & & 5 & 2 & 8 & 7 & \\
\hline
\end{tabular}

$d_{2}^{* H L}=(34.57692,0.559017), d_{2}^{-H L}=(0,0.7071067812)$.

Now, it is easy to compute step (10):

Maximize $\delta^{H L-D M_{L_{1}}}$

Subject to

$3 x_{1}+5 x_{2}+x_{3} \leq 35,2 x_{1}-x_{2}+12 x_{3} \leq 20,5 x_{2}+6 x_{3} \leq$ $16, x_{1} \geq 1, x_{1}, x_{2}, x_{3} \geq 0$

$\left(\frac{d_{1}^{P I S}{ }^{H L}(X)-34.57692}{0.5590170-34.57692}\right) \geq \delta^{H L-D M_{L_{1}}}$

$\left(\frac{{\text { zero }-d_{1}^{N I S}}^{H L}(X)}{\text { zero-0.7071067812 }}\right) \geq \delta^{H L-D M_{L_{1}}}, \delta^{H L-D M_{L_{1}}} \in[0,1]$.

The maximum "satisfactory level" ( $\left.\delta^{H L-D M_{L_{1}}}=0.1536454 \mathrm{E}-01\right)$ is achieved for the solution $x_{1}^{* H L}=10.38462, x_{2}^{* H L}=0.7692308, x_{3}^{* H L}=$ zero.

(Second Level):

Obtain PIS and NIS payoff tables for problem $P^{H L}$ :

Table 13. PIS payoff table for problem $P^{H L}$

\begin{tabular}{lccccc}
\hline & $\boldsymbol{F}_{\mathbf{2 1}}^{\boldsymbol{H}}(\boldsymbol{X})$ & $\boldsymbol{F}_{\mathbf{2 2}}^{\boldsymbol{H L}}(\boldsymbol{X})$ & $\boldsymbol{x}_{\mathbf{1}}$ & $\boldsymbol{x}_{\mathbf{2}}$ & $\boldsymbol{x}_{\mathbf{3}}$ \\
\hline $\operatorname{Max} . F_{21}^{H L}(X)$ & $39.23^{*}$ & 3.56 & 7.4 & 2.44 & 0.64 \\
$\operatorname{Max} . F_{22}^{H L}(X)$ & 26 & $14^{*}$ & 10 & 0 & 0 \\
\hline
\end{tabular}

PIS: $f^{* H L}=(39.2258,14)$

Table 14. NIS payoff table for problem $P^{H L}$

\begin{tabular}{lccccc}
\hline & $\boldsymbol{F}_{\mathbf{2 1}}^{H L}(\boldsymbol{X})$ & $\boldsymbol{F}_{\mathbf{2 2}}^{H L}(\boldsymbol{X})$ & $\boldsymbol{x}_{\mathbf{1}}$ & $\boldsymbol{x}_{\mathbf{2}}$ & $\boldsymbol{x}_{\mathbf{3}}$ \\
\hline $\operatorname{Min} F_{21}^{H L}(X)$ & $6^{-}$ & 4 & 0 & 0 & 0 \\
$\operatorname{Min} F_{22}^{H L}(X)$ & 25.2 & $-8.8^{-}$ & 0 & 3.2 & 0 \\
\hline
\end{tabular}

$\mathrm{NIS}: f^{-H L}=(6,-8.8)$
Next, construct equation and obtain the following equations:

$$
\begin{gathered}
d_{p}^{P I S^{H L-D M_{L}}}=\left[w_{1}^{p}\left(\frac{69.15385-F_{11}^{H L}(X)}{69.15385-3}\right)^{p}+\right. \\
w_{2}^{p}\left(\frac{74.92308-F_{12}^{H L}(X)}{74.92308-(-0.2)}\right)^{p}+w_{3}^{p}\left(\frac{39.2258-F_{21}^{H L}(X)}{39.2258-6}\right)^{p}+ \\
\left.w_{4}^{p}\left(\frac{14-F_{22}^{H L}(X)}{14-(-8.8)}\right)^{p}\right]^{1 / p} \\
d_{p}^{N I S^{H L-D M_{L 2}}}=\left[w_{1}^{p}\left(\frac{F_{11}^{H L}(X)-(3)}{69.15385-3}\right)^{p}+w_{2}^{p}\left(\frac{F_{12}^{H L}(X)-(-0.2)}{74.92308-(-0.2)}\right)^{p}+\right. \\
\left.w_{3}^{p}\left(\frac{F_{21}^{H L}(X)-(6)}{39.2258-6}\right)^{p}+w_{4}^{p}\left(\frac{F_{22}^{H L}(X)-(-8.8)}{14-(-8.8)}\right)^{p}\right]^{1 / p}
\end{gathered}
$$

Thus, problem (11) is obtained. In order to get numerical solutions, assume that $w_{1}^{p}=w_{2}^{p}=w_{3}^{p}=w_{4}^{p}=0.25$ and $\mathrm{p}=2$,

Table 15. PIS payoff table of problem (11) when $\mathrm{p}=2$

\begin{tabular}{lccccccc}
\hline & $\boldsymbol{d}_{\mathbf{2}}^{\text {PIS }}$ & $\boldsymbol{d}_{\mathbf{2}}^{\text {NIS }}$ & $\boldsymbol{F}_{\mathbf{2 1}}^{H L}$ & $\boldsymbol{F}_{\mathbf{2 2}}^{H L}$ & $\boldsymbol{x}_{\mathbf{1}}$ & $\boldsymbol{x}_{\mathbf{2}}$ & $\boldsymbol{x}_{\mathbf{3}}$ \\
\hline Min. $d_{2}^{\text {PIS }}$ & $9.5^{*}$ & $0.6^{-}$ & 39. & 3.6 & 7.4 & 2.4 & 0.6 \\
& & & 2 & & & 4 & 4 \\
Max. $_{2}^{N I S^{L L}}$ & 0.1 & $0.2^{*}$ & 31. & 11. & 10. & 0.7 & 0 \\
& & & 4 & 3 & 4 & 7 & \\
\hline
\end{tabular}

$d_{2}^{* H L}=(9.511326,0.1645195), d_{2}^{-H L}=(0.06597257621$, $0.5708198897)$.

Now, it is easy to compute step (10):

Maximize $\delta^{H L-D M_{L_{2}}}$

Subject to

$$
\begin{gathered}
3 x_{1}+5 x_{2}+x_{3} \leq 35,2 x_{1}-x_{2}+12 x_{3} \leq 20,5 x_{2}+6 x_{3} \leq \\
16, x_{1} \geq 1, x_{1}, x_{2}, x_{3} \geq 0
\end{gathered}
$$

$\left(\frac{d_{1}^{P I S^{H L}}(X)-9.511326}{0.1645195-9.511326}\right) \geq$

$\delta^{H L-D M_{L_{2}}},\left(\frac{0.06597257621-d_{1}^{N I S^{H L}}(X)}{0.06597257621-0.5708198897}\right) \geq \delta^{H L-D M_{L_{2}}}$,

$\delta^{H L-D M_{L_{2}}} \in[0,1]$

$\left(\frac{(10.38462+0.001)-x_{1}}{0.001}\right) \geq \delta^{H L-D M_{L_{2}}},\left(\frac{x_{1}-(10.38462-0.001)}{0.001}\right) \geq$

The maximum "satisfactory level" $\left(\delta^{H L-D M_{L_{2}}}=\right.$ zero $)$ is achieved for the solution $x_{1}^{* H L}=10.38462$, $x_{2}^{*^{H L}}=0.7692308, x_{3}^{* H L}=0.2121311 \mathrm{e}-03$.

(Third Level):

Obtain PIS and NIS payoff tables for problem $P^{H L}$ :

Table 16. PIS payoff table for problem $P^{H L}$

\begin{tabular}{lccccc}
\hline & $\boldsymbol{F}_{\mathbf{3 1}}^{\boldsymbol{H}}(\boldsymbol{X})$ & $\boldsymbol{F}_{\mathbf{3 2}}^{\boldsymbol{H}}(\boldsymbol{X})$ & $\boldsymbol{x}_{\mathbf{1}}$ & $\boldsymbol{x}_{\mathbf{2}}$ & $\boldsymbol{x}_{\mathbf{3}}$ \\
\hline $\operatorname{Max} . F_{31}^{H L}(X)$ & $55.38^{*}$ & 62 & 10.38 & 0.77 & 0 \\
$\operatorname{Max} . F_{32}^{H L}(X)$ & 55.38 & $62^{*}$ & 10.38 & 0.77 & 0 \\
\hline
\end{tabular}

PIS: $f^{*^{H L}}=(55.38462,62)$ 
Table 17. NIS payoff table for problem $P^{H L}$

\begin{tabular}{lccccc}
\hline & $\boldsymbol{F}_{\mathbf{3 1}}^{\boldsymbol{H}}(\boldsymbol{X})$ & $\boldsymbol{F}_{\mathbf{3 2}}^{\boldsymbol{H}}(\boldsymbol{X})$ & $\boldsymbol{x}_{\mathbf{1}}$ & $\boldsymbol{x}_{\mathbf{2}}$ & $\boldsymbol{x}_{\mathbf{3}}$ \\
\hline $\operatorname{Min} F_{31}^{H L}(X)$ & $-1.4^{-}$ & 19.8 & 0 & 3.2 & 0 \\
$\operatorname{Min} . F_{32}^{H L}(X)$ & 6.67 & $5.33^{-}$ & 0 & 0 & 1.67 \\
\hline
\end{tabular}

NIS: $f^{-H L}=(-1.4,5.3333)$

Next, construct equation and obtain the following equations:

$$
\begin{aligned}
d_{p}^{P I S}{ }^{H L-D M_{L_{3}}}=\left[w_{1}^{p}\left(\frac{69.15385-F_{11}^{H L}(X)}{69.15385-3}\right)^{p}+\right. \\
w_{2}^{p}\left(\frac{74.92308-F_{12}^{H L}(X)}{74.92308-(-0.2)}\right)^{p}+w_{3}^{p}\left(\frac{39.2258-F_{21}^{H L}(X)}{39.2258-6}\right)^{p}+ \\
w_{4}^{p}\left(\frac{14-F_{22}^{H L}(X)}{14-(-8.8)}\right)^{p}+w_{5}^{p}\left(\frac{55.38462-F_{31}^{H L}(X)}{55.38462-(-1.4)}\right)^{p}+ \\
\\
\left.w_{6}^{p}\left(\frac{62-F_{32}^{H L}(X)}{62-(5.3333)}\right)^{p}\right]^{1 / p}
\end{aligned}
$$

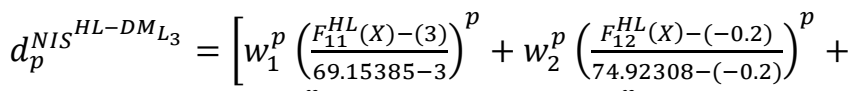$$
w_{3}^{p}\left(\frac{F_{21}^{H L}(X)-(6)}{39.2258-6}\right)^{p}+w_{4}^{p}\left(\frac{F_{22}^{H L}(X)-(-8.8)}{14-(-8.8)}\right)^{p}+
$$$$
\left.w_{5}^{p}\left(\frac{F_{31}^{H L}(X)-(-1.4)}{55.38462-(-1.4)}\right)^{p}+w_{6}^{p}\left(\frac{F_{32}^{H L}(X)-(5.3333)}{62-(5.33333)}\right)^{p}\right]^{1 / p}
$$

\begin{tabular}{|c|c|c|c|c|c|c|c|}
\hline & $d_{3}^{P I S^{H L}}$ & $d_{3}^{N I S^{H L}}$ & $F_{31}^{H L}(X)$ & $F_{32}^{H L}(X)$ & $x_{1}$ & $x_{2}$ & $x_{3}$ \\
\hline Min. $d_{3}^{P I S^{L L}}$ & $9.1^{*}$ & $0.4^{-}$ & 55.4 & 62 & $\begin{array}{c}10 . \\
4\end{array}$ & $\begin{array}{c}0.7 \\
7\end{array}$ & 0 \\
\hline $\operatorname{Max} . d_{3}^{N I S^{L I}}$ & 0.04 & $0.1^{*}$ & 55.4 & 62 & $\begin{array}{c}10 . \\
4\end{array}$ & $\begin{array}{c}0.7 \\
7\end{array}$ & 0 \\
\hline
\end{tabular}

Thus, problem (11) is obtained. In order to get numerical solutions, assume that $w_{1}^{p}=w_{2}^{p}=w_{3}^{p}=w_{4}^{p}=w_{5}^{p}=w_{6}^{p}=1 / 6$ and $\mathrm{p}=2$,

Table 18. PIS payoff table of problem (11) when $\mathrm{p}=2$

$d_{3}^{*^{H L}}=(9.068215,0.1009190), d_{3}^{-H L}=(0.04398171747$, $0.3859144257)$.

Now, it is easy to compute step (10):

Maximize $\delta^{H L-D M_{L_{3}}}$

Subject to

$3 x_{1}+5 x_{2}+x_{3} \leq 35,2 x_{1}-x_{2}+12 x_{3} \leq 20,5 x_{2}+6 x_{3} \leq$ $16, x_{1} \geq 1, x_{1}, x_{2}, x_{3} \geq 0$

$\left(\frac{d_{3}^{P I S^{H L}}(X)-9.068215}{0.1009190-9.068215}\right) \geq \delta^{H L-D M_{L_{3}}}$,

$\left(\frac{0.04398171747-d_{3}^{N I S^{H L}}(X)}{0.04398171747-0.3859144257}\right) \geq \delta^{H L-D M_{L_{3}}}$

$\delta^{H L-D M_{L_{3}}} \in[0,1]$.

$\left(\frac{(0+0.001)-x_{3}}{0.001}\right) \geq \delta^{H L-D M_{L_{3}}},\left(\frac{x_{3}-(0-0.001)}{0.001}\right) \geq \delta^{H L-D M_{L_{3}}}$

The maximum "satisfactory level" $\left(\delta^{H L-D M_{L_{3}}}=\right.$ zero $)$ is achieved for the solution $x_{1}^{* H L}=10.38462$, $x_{2}^{*^{H L}}=0.7692308, x_{3}^{* H L}=$ zero.

\section{(First Level):}

$\underline{P^{L H}:}$

Obtain PIS and NIS payoff tables for problem $P^{L H}$ :

Table 19. PIS payoff table for problem $P^{L H}$

\begin{tabular}{lccccc}
\hline & $\boldsymbol{F}_{11}^{\mathbf{L H}}(\boldsymbol{X})$ & $\boldsymbol{F}_{\mathbf{1 2}}^{\mathbf{L H}}(\boldsymbol{X})$ & $\boldsymbol{x}_{\mathbf{1}}$ & $\boldsymbol{x}_{\mathbf{2}}$ & $\boldsymbol{x}_{\mathbf{3}}$ \\
\hline $\operatorname{Max} . F_{11}^{L H}(X)$ & $23.31^{*}$ & 51.15 & 10.38 & 0.77 & 0 \\
$\operatorname{Max} . F_{12}^{L H}(X)$ & 23.31 & $51.15^{*}$ & 10.38 & 0.77 & 0 \\
\hline
\end{tabular}

PIS: $f^{*^{L H}}=(23.30769,51.15385)$

Table 20. NIS payoff table for problem $P^{L H}$

\begin{tabular}{lccccc}
\hline & $\boldsymbol{F}_{1}^{\boldsymbol{L H}}(\boldsymbol{X})$ & $\boldsymbol{F}_{\mathbf{2}}^{\boldsymbol{L H}}(\boldsymbol{X})$ & $\boldsymbol{x}_{\mathbf{1}}$ & $\boldsymbol{x}_{\mathbf{2}}$ & $\boldsymbol{x}_{\mathbf{3}}$ \\
\hline $\operatorname{Min} F_{11}^{L H}(X)$ & $1^{-}$ & 0 & 0 & 0 & 0 \\
$\operatorname{Min} F_{12}^{L H}(X)$ & 7.4 & $-3.2^{-}$ & 0 & 3.2 & 0 \\
\hline
\end{tabular}

NIS: $f^{-L H}=(1,-3.2)$

Next, construct equation and obtain the following equations:

$$
\begin{gathered}
d_{p}^{P I S^{L H-D M_{L_{1}}}}=\left[w_{1}^{p}\left(\frac{23.30769-F_{11}^{L H}(X)}{23.30769-1}\right)^{p}+\right. \\
\left.w_{2}^{p}\left(\frac{51.15385-F_{12}^{L H}(X)}{51.15385-(-3.2)}\right)^{p}\right]^{1 / p}
\end{gathered}
$$

\begin{tabular}{|c|c|c|c|c|c|c|c|}
\hline & $d_{2}^{P I S^{L H}}$ & $d_{2}^{N I S^{L H}}$ & $F_{11}^{L H}(X)$ & $F_{12}^{L H}(X)$ & $x_{1}$ & $x_{2}$ & $x_{3}$ \\
\hline Min. $d_{2}^{P I S^{L H}}$ & $11.13^{*}$ & $0.71^{-}$ & 23.31 & 51.2 & $\begin{array}{c}10 . \\
4\end{array}$ & $\begin{array}{c}0 . \\
8\end{array}$ & 0 \\
\hline $\operatorname{Max} . d_{2}^{N I S^{L H}}$ & 0 & $0.56^{*}$ & 23.31 & 51.2 & $\begin{array}{c}10 . \\
4\end{array}$ & $\begin{array}{l}0 . \\
8\end{array}$ & 0 \\
\hline
\end{tabular}

$$
\begin{gathered}
d_{p}^{N I S^{L H-D M_{L_{1}}}}=\left[w_{1}^{p}\left(\frac{F_{11}^{L H}(X)-(1)}{23.30769-1}\right)^{p}+\right. \\
\left.w_{2}^{p}\left(\frac{F_{12}^{L H}(X)-(-3.2)}{51.15385-(-3.2)}\right)^{p}\right]^{1 / p}
\end{gathered}
$$

Thus, problem (11) is obtained. In order to get numerical solutions, assume that $w_{1}^{p}=w_{2}^{p}=0.5$ and $\mathrm{p}=2$,

Table 21. PIS payoff table of problem (11) when $\mathrm{p}=2$

$d_{2}^{*^{L H}}=(11.13143,0.5590170), d_{2}^{-L H}=(0,0.7071067812)$.

Now, it is easy to compute step (10):

Maximize $\delta^{L H-D M_{L_{1}}}$

Subject to

$$
\begin{aligned}
& 3 x_{1}+5 x_{2}+x_{3} \leq 35,2 x_{1}-x_{2}+12 x_{3} \leq 20,5 x_{2}+6 x_{3} \leq \\
& 16, x_{1} \geq 1, x_{1}, x_{2}, x_{3} \geq 0 \\
& \left(\frac{d_{1}^{P I S^{L H}}(X)-11.13143}{0.5590170-11.13143}\right) \geq \delta^{L H-D M_{L_{1}}} \\
& \left(\frac{\text { zero- } d_{1}^{N I S^{L H}}(X)}{\text { zero-0.7071067812 }}\right) \geq \delta^{L H-D M_{L_{1}}, \delta^{L H-D M_{L_{1}}} \in[0,1] .}
\end{aligned}
$$

The maximum "satisfactory level" 
( $\left.\delta^{L H-D M_{L_{1}}}=0.1536454 \mathrm{E}-01\right)$ is achieved for the solution $x_{1}^{* L H}=10.38462, x_{2}^{* L H}=0.7692308, x_{3}^{* L H}=$ zero.

(Second Level):

Obtain PIS and NIS payoff tables for problem $P^{L H}$ :

Table 22. PIS payoff table for problem $P^{L H}$

\begin{tabular}{lccccc}
\hline & $\boldsymbol{F}_{\mathbf{2 1}}^{\mathbf{L H}}(\boldsymbol{X})$ & $\boldsymbol{F}_{\mathbf{2 2}}^{\mathbf{L H}}(\boldsymbol{X})$ & $\boldsymbol{x}_{\mathbf{1}}$ & $\boldsymbol{x}_{\mathbf{2}}$ & $\boldsymbol{x}_{\mathbf{3}}$ \\
\hline $\operatorname{Max} . F_{21}^{L H}(X)$ & $26.08^{*}$ & 10.85 & 10.38 & 0.77 & 0 \\
$\operatorname{Max} . F_{22}^{L H}(X)$ & 23 & $12^{*}$ & 10 & 0 & 0 \\
\hline
\end{tabular}

PIS: $f^{*^{L H}}=(26.07692,12)$

Table 23. NIS payoff table for problem $P^{L H}$

\begin{tabular}{lccccc}
\hline & $\boldsymbol{F}_{\mathbf{2 1}}^{\mathbf{L H}}(\boldsymbol{X})$ & $\boldsymbol{F}_{\mathbf{2 2}}^{\boldsymbol{L H}}(\boldsymbol{X})$ & $\boldsymbol{x}_{\mathbf{1}}$ & $\boldsymbol{x}_{\mathbf{2}}$ & $\boldsymbol{x}_{\mathbf{3}}$ \\
\hline $\operatorname{Min} F_{21}^{L H}(X)$ & $3^{-}$ & 2 & 0 & 0 & 0 \\
$\operatorname{Min} F_{22}^{L H}(X)$ & 12.6 & $-4.4^{-}$ & 0 & 3.2 & 0 \\
\hline
\end{tabular}

$\mathrm{NIS}: f^{-^{L H}}=(3,-4.4)$

Next, construct equation and obtain the following equations:

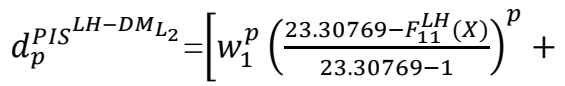

$$
\begin{aligned}
& w_{2}^{p}\left(\frac{51.15385-F_{12}^{L H}(X)}{51.15385-(-3.2)}\right)^{p}+w_{3}^{p}\left(\frac{26.07692-F_{21}^{L H}(X)}{26.07692-3}\right)^{p}+ \\
& \left.w_{4}^{p}\left(\frac{12-F_{22}^{L H}(X)}{12-(-4.4)}\right)^{p}\right]^{1 / p}
\end{aligned}
$$$$
d_{p}^{N I S^{L H-D M_{L_{2}}}=}
$$$$
\left[w_{1}^{p}\left(\frac{F_{11}^{H L}(X)-(1)}{23.30769-1}\right)^{p}+w_{2}^{p}\left(\frac{F_{12}^{H L}(X)-(-3.2)}{51.15385-(-3.2)}\right)^{p}+\right.
$$$$
\left.w_{3}^{p}\left(\frac{F_{21}^{H L}(X)-(3)}{26.07692-3}\right)^{p}+w_{4}^{p}\left(\frac{F_{22}^{H L}(X)-(-4.4)}{12-(-4.4)}\right)^{p}\right]^{1 / p}
$$

Thus, problem (11) is obtained. In order to get numerical solutions, assume that $w_{1}^{p}=w_{2}^{p}=w_{3}^{p}=w_{4}^{p}=0.25$ and $\mathrm{p}=2$,

Table 24. PIS payoff table of problem (11) when $\mathrm{p}=2$

\begin{tabular}{lccccccc}
\hline & $\boldsymbol{d}_{\mathbf{2}}^{P I S^{L H}}$ & $\boldsymbol{d}_{\mathbf{2}}^{\text {NIS }}$ & $\boldsymbol{F}_{\mathbf{2 1}}^{L \boldsymbol{L}}(\boldsymbol{X})$ & $\boldsymbol{F}_{\mathbf{2 2}}^{L \boldsymbol{H}}(\boldsymbol{X})$ & $\boldsymbol{x}_{\mathbf{1}}$ & $\boldsymbol{x}_{\mathbf{2}}$ & $\boldsymbol{x}_{\mathbf{3}}$ \\
\hline Min. $d_{2}^{P I S^{L L}}$ & $6.24^{*}$ & $0.49^{-}$ & 26.1 & 10.9 & 10. & 0. & 0 \\
& & & & & 4 & 8 & \\
Max. $d_{2}^{N I S^{L L}}$ & 0.03 & $0.19^{*}$ & 26.1 & 10.9 & 10. & 0. & 0 \\
& & & & & 4 & 8 & \\
\hline
\end{tabular}

$d_{2}^{*^{L H}}=(6.236730,0.1908760), d_{2}^{-L H}=(0.02462469512$, $0.4914416041)$.

Now, it is easy to compute step (10):

Maximize $\delta^{L H-D M_{L_{2}}}$

Subject to

$$
\begin{gathered}
3 x_{1}+5 x_{2}+x_{3} \leq 35,2 x_{1}-x_{2}+12 x_{3} \leq 20,5 x_{2}+6 x_{3} \leq \\
16, x_{1} \geq 1, x_{1}, x_{2}, x_{3} \geq 0
\end{gathered}
$$

$\left(\frac{d_{1}^{P I S^{L H}}(X)-6.236730}{0.1908760-6.236730}\right) \geq \delta^{L H-D M_{L_{2}}}$,

$\left(\frac{0.02462469512-d_{1}^{N I S^{L H}}(X)}{0.02462469512-0.4914416041}\right) \geq \delta^{L H-D M_{L_{2}}}$,

$$
\delta^{L H-D M_{L_{2}}} \in[0,1]
$$

$\left(\frac{(10.38462+0.001)-x_{1}}{0.001}\right) \geq \delta^{L H-D M_{L_{2}}}$

$\left(\frac{x_{1}-(10.38462-0.001)}{0.001}\right) \geq \delta^{L H-D M_{L_{2}}}$

The maximum "satisfactory level" $\left(\delta^{L H-D M_{L_{2}}}=3561380\right)$ is achieved for the solution $x_{1}^{*}=10.38462$, $x_{2}^{* L H}=0.7692308, x_{3}^{* L H}=$ zero.

\section{(Third Level):}

Obtain PIS and NIS payoff tables for problem $P^{L H}$ :

Table 25. PIS payoff table for problem $P^{L H}$

\begin{tabular}{lccccc}
\hline & $\boldsymbol{F}_{\mathbf{3 1}}^{\mathbf{L H}}(\boldsymbol{X})$ & $\boldsymbol{F}_{\mathbf{3 2}}^{\mathbf{L H}}(\boldsymbol{X})$ & $\boldsymbol{x}_{\mathbf{1}}$ & $\boldsymbol{x}_{\mathbf{2}}$ & $\boldsymbol{x}_{\mathbf{3}}$ \\
\hline $\operatorname{Max} . F_{31}^{H L}(X)$ & $13^{*}$ & 55 & 10 & 0 & 0 \\
$\operatorname{Max} . F_{32}^{L H}(X)$ & 11.85 & $58.46^{*}$ & 10.38 & 0.77 & 0 \\
\hline
\end{tabular}

PIS: $f^{*^{L H}}=(13,58.46154)$

Table 26. NIS payoff table for problem $P^{L H}$

\begin{tabular}{lccccc}
\hline & $\boldsymbol{F}_{\mathbf{3 1}}^{\mathbf{L H}}(\boldsymbol{X})$ & $\boldsymbol{F}_{\mathbf{3 2}}^{\mathbf{L H}}(\boldsymbol{X})$ & $\boldsymbol{x}_{\mathbf{1}}$ & $\boldsymbol{x}_{\mathbf{2}}$ & $\boldsymbol{x}_{\mathbf{3}}$ \\
\hline Min. $F_{31}^{\mathbf{L H}}(X)$ & $-3.4^{-}$ & 11.4 & 0 & 3.2 & 0 \\
$\operatorname{Min} F_{32}^{L H}(X)$ & 4.67 & $3.33^{-}$ & 0 & zero & 1.67 \\
\hline
\end{tabular}

NIS: $f^{-{ }^{L H}}=(-3.4,3.3333)$

Next, construct equation and obtain the following equations:

$$
\begin{aligned}
& d_{p}^{P I S^{L H-D M_{L 3}}}=\left[w_{1}^{p}\left(\frac{23.30769-F_{11}^{L H}(X)}{23.30769-1}\right)^{p}+\right. \\
& w_{2}^{p}\left(\frac{51.15385-F_{12}^{L H}(X)}{51.15385-(-3.2)}\right)^{p}+w_{3}^{p}\left(\frac{26.07692-F_{21}^{L H}(X)}{26.07692-3}\right)^{p}+ \\
& w_{4}^{p}\left(\frac{12-F_{22}^{L H}(X)}{12-(-4.4)}\right)^{p}+w_{5}^{p}\left(\frac{13-F_{31}^{L H}(X)}{13-(-3.4)}\right)^{p}+ \\
& \left.w_{6}^{p}\left(\frac{58.46154-F_{32}^{L H}(X)}{58.46154-(3.3333)}\right)^{p}\right]^{1 / p}
\end{aligned}
$$

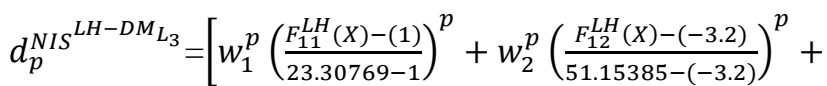

$$
\begin{aligned}
& w_{3}^{p}\left(\frac{F_{21}^{L H}(X)-(3)}{26.07692-3}\right)^{p}+w_{4}^{p}\left(\frac{F_{22}^{L H}(X)-(-4.4)}{12-(-4.4)}\right)^{p}+ \\
& \left.w_{5}^{p}\left(\frac{F_{31}^{L H}(X)-(-3.4)}{13-(-3.4)}\right)^{p}+w_{6}^{p}\left(\frac{F_{32}^{L H}(X)-(3.3333)}{58.46154-(3.33333)}\right)^{p}\right]^{1 / p}
\end{aligned}
$$

Thus, problem (11) is obtained. In order to get numerical solutions, assume that $w_{1}^{p}=w_{2}^{p}=w_{3}^{p}=w_{4}^{p}=w_{5}^{p}=w_{6}^{p}=1 / 6$ and $\mathrm{p}=2$, 
Table 27. PIS payoff table of problem (11) when $\mathrm{p}=2$

\begin{tabular}{lccccccc}
\hline & $d_{3}^{\text {PIS }}$ & $d_{3}^{\text {NIS }}$ & $F_{31}^{L H}(X)$ & $F_{32}^{L H}(X)$ & $x_{1}$ & $x_{2}$ & $x_{3}$ \\
\hline Min. $d_{3}^{P I S^{L L}}$ & $2.03^{*}$ & $0.4^{-}$ & 13 & 55 & 10 & 0 & 0 \\
Max.d & & & & & & & \\
3 & 0.02 & $0 . S^{L L}$ & 11.85 & 58.46 & 10. & 0.7 & 0 \\
\hline
\end{tabular}

$d_{3}^{*^{L H}}=(2.034558, .1087784), d_{3}^{-L H}=(0.01658311422$, $0.386786411)$.

Now, it is easy to compute step (10):

Maximize $\delta^{L H-D M_{L_{3}}}$

Subject to

$3 x_{1}+5 x_{2}+x_{3} \leq 35,2 x_{1}-x_{2}+12 x_{3} \leq 20,5 x_{2}+6 x_{3} \leq$ $16, x_{1} \geq 1, x_{1}, x_{2}, x_{3} \geq 0$

$\left(\frac{d_{3}^{P I S^{L H}}(X)-2.034558}{0.1087784-2.034558}\right) \geq \delta^{L H-D M_{L_{3}}}$

$\left(\frac{0.01658311422-d_{3}^{N I} S^{L H}(X)}{0.01658311422-0.386786411}\right) \geq \delta^{L H-D M_{L_{3}}}$,

$\delta^{L H-D M_{L_{3}}} \in[0,1]$.

$\left(\frac{(0+0.001)-x_{3}}{0.001}\right) \geq \delta^{L H-D M_{L_{3}}},\left(\frac{x_{3}-(0-0.001)}{0.001}\right) \geq \delta^{L H-D M_{L_{3}}}$

The maximum "satisfactory level" $\left(\delta^{L H-D M_{L_{3}}}=\right.$ zero $)$ is achieved for the solution $x_{1}^{*^{L H}}=10, x_{2}^{* L H}=$ zero, $x_{3}^{*^{L H}}=$ zero.

(First Level):

$$
\underline{P^{H H}:}
$$

Obtain PIS and NIS payoff tables for problem $P^{H H}$ :

Table 28. PIS payoff table for problem $P^{H H}$

\begin{tabular}{lccccc}
\hline & $\boldsymbol{F}_{\mathbf{1 1}}^{\boldsymbol{H}}(\boldsymbol{X})$ & $\boldsymbol{F}_{\mathbf{1 2}}^{\boldsymbol{H H}}(\boldsymbol{X})$ & $\boldsymbol{x}_{\mathbf{1}}$ & $\boldsymbol{x}_{\mathbf{2}}$ & $\boldsymbol{x}_{\mathbf{3}}$ \\
\hline $\operatorname{Max} . F_{11}^{H H}(X)$ & $113.23^{*}$ & 97.69 & 10.4 & 0.77 & 0 \\
$\operatorname{Max} . F_{12}^{H H}(X)$ & 113.23 & $97.69^{*}$ & 10.4 & 0.77 & 0 \\
\hline
\end{tabular}

PIS: $f^{*{ }^{H H}}=(113.2308,97.69231)$

Table 29. NIS payoff table for problem $P^{H H}$

\begin{tabular}{lccccc}
\hline & $\boldsymbol{F}_{\mathbf{1}}^{\boldsymbol{H}}(\boldsymbol{X})$ & $\boldsymbol{F}_{\mathbf{2}}^{\boldsymbol{H}}(\boldsymbol{X})$ & $\boldsymbol{x}_{\mathbf{1}}$ & $\boldsymbol{x}_{\mathbf{2}}$ & $\boldsymbol{x}_{\mathbf{3}}$ \\
\hline $\operatorname{Min} . F_{11}^{H H}(X)$ & $4^{-}$ & 5 & 0 & 0 & 0 \\
$\operatorname{Min} . F_{12}^{H H}(X)$ & 26.4 & $1.8^{-}$ & 0 & 3.2 & 0 \\
\hline
\end{tabular}

NIS: $f^{-{ }^{H H}}=(4,1.8)$

Next, construct equation and obtain the following equations:

$$
\begin{aligned}
& d_{p}^{P I S^{H H-D M_{L_{1}}}}=\left[w_{1}^{p}\left(\frac{113.2308-F_{11}^{H H}(X)}{113.2308-4}\right)^{p}+\right. \\
& \left.w_{2}^{p}\left(\frac{97.69231-F_{12}^{H H}(X)}{97.69231-(1.8)}\right)^{p}\right]^{1 / p}
\end{aligned}
$$

$d_{p}^{N I S^{H H-D M_{L_{1}}}}=\left[w_{1}^{p}\left(\frac{F_{11}^{H H}(X)-(4)}{113.2308-4}\right)^{p}+w_{2}^{p}\left(\frac{F_{12}^{H H}(X)-(1.8)}{97.69231-(1.8)}\right)^{p}\right]^{1 / p}$
Thus, problem (11) is obtained. In order to get numerical

\begin{tabular}{|c|c|c|c|c|c|c|c|}
\hline & $d_{2}^{P I S^{H H}}$ & $d_{2}^{N I S^{H H}}$ & $F_{11}^{H H}(X)$ & $F_{12}^{H H}(X)$ & $x_{1}$ & $x_{2}$ & $x_{3}$ \\
\hline Min. $d_{2}^{P I S^{H H}}$ & $56.1^{*}$ & $0.71^{-}$ & 113.23 & 97.69 & $\begin{array}{c}10 . \\
4\end{array}$ & $\begin{array}{l}0 \\
8\end{array}$ & 0 \\
\hline $\operatorname{Max} \cdot d_{2}^{N I S^{H F}}$ & 0 & $0.56^{*}$ & 113.23 & 97.691 & $\begin{array}{c}10 . \\
4\end{array}$ & $\begin{array}{l}0 . \\
8\end{array}$ & 0 \\
\hline
\end{tabular}
solutions, assume that $w_{1}^{p}=w_{2}^{p}=0.5$ and $\mathrm{p}=2$.

Table 30. PIS payoff table of problem (11) when $\mathrm{p}=2$

Now, it is easy to compute step (10):

Maximize $\delta^{H H-D M_{L_{1}}}$

Subject to

$$
\begin{aligned}
& 3 x_{1}+5 x_{2}+x_{3} \leq 35,2 x_{1}-x_{2}+12 x_{3} \leq 20,5 x_{2}+6 x_{3} \leq \\
& 16, x_{1} \geq 1, x_{1}, x_{2}, x_{3} \geq 0 \\
& \left(\frac{d_{1}^{P I S} S^{H H}}{0.5590169-56.09709}\right) \geq \delta^{H H-D M_{L_{1}}}, \\
& \left(\frac{\text { zero- } d_{1}^{N I S} S^{H H}}{\text { zero }-0.707109^{H}}\right) \geq \delta^{H H-D M_{L_{1}}}, \\
& \delta^{H H-D M_{L_{1}}} \in[0,1] .
\end{aligned}
$$

The maximum "satisfactory level" $\left(\delta^{H H-D M_{L_{1}}}=\right.$ zero $)$ is achieved for the solution $x_{1}^{{ }^{*}{ }^{H H}}=10.38462$, $x_{2}^{* H H}=0.7692308, x_{3}^{* H H}=$ zero.

\section{(Second Level):}

Obtain PIS and NIS payoff tables for problem $P^{H H}$ :

Table 31. PIS payoff table for problem $P^{H H}$

\begin{tabular}{lccccc}
\hline & $\boldsymbol{F}_{\mathbf{2 1}}^{\boldsymbol{H}}(\boldsymbol{X})$ & $\boldsymbol{F}_{\mathbf{2 2}}^{\boldsymbol{H}}(\boldsymbol{X})$ & $\boldsymbol{x}_{\mathbf{1}}$ & $\boldsymbol{x}_{\mathbf{2}}$ & $\boldsymbol{x}_{\mathbf{3}}$ \\
\hline $\operatorname{Max} . F_{21}^{H H}(X)$ & $45.27^{*}$ & -6.87 & 6.3 & 3.2 & 0 \\
$\operatorname{Max} . F_{22}^{H H}(X)$ & -13 & $17.67^{*}$ & 0 & 0 & 1.67 \\
\hline
\end{tabular}

PIS: $f^{* H H}=(45.26667,17.66667)$

Table 32. NIS payoff table for problem $P^{H H}$

\begin{tabular}{lccccc}
\hline & $\boldsymbol{F}_{\mathbf{2 1}}^{\boldsymbol{H}}(\boldsymbol{X})$ & $\boldsymbol{F}_{\mathbf{2 2}}^{\boldsymbol{H}}(\boldsymbol{X})$ & $\boldsymbol{x}_{\mathbf{1}}$ & $\boldsymbol{x}_{\mathbf{2}}$ & $\boldsymbol{x}_{\mathbf{3}}$ \\
\hline $\operatorname{Min}_{\operatorname{Min} F_{21}^{H H}(X)}$ & $-13^{-}$ & 17.67 & 0 & 0 & 1.67 \\
$\operatorname{Min} F_{22}^{H H}(X)$ & 32.6 & $-13.2^{-}$ & 0 & 3.2 & 0 \\
\hline
\end{tabular}

NIS: $f^{-H L}=(-13,-13.2)$

Next, construct equation and obtain the following equations:

$$
\begin{aligned}
& d_{p}^{P I S}{ }^{H H-D M_{L_{2}}}=\left[w_{1}^{p}\left(\frac{113.2308-F_{11}^{H H}(X)}{113.2308-4}\right)^{p}+\right. \\
& w_{2}^{p}\left(\frac{97.69231-F_{12}^{H H}(X)}{97.69231-(1.8)}\right)^{p}+w_{3}^{p}\left(\frac{45.26667-F_{21}^{H H}(X)}{45.26667-(-13)}\right)^{p}+ \\
& \left.w_{4}^{p}\left(\frac{17.66667-F_{22}^{H H}(X)}{17.66667-(-13.2)}\right)^{p}\right]^{1 / p}
\end{aligned}
$$




$$
\begin{aligned}
& d_{p}^{N I S^{H H-D M_{L_{2}}}}=\left[w_{1}^{p}\left(\frac{F_{11}^{H H}(X)-(4)}{113.2308-4}\right)^{p}+w_{2}^{p}\left(\frac{F_{12}^{H H}(X)-(1.8)}{97.69231-(1.8)}\right)^{p}+\right. \\
& \left.w_{3}^{p}\left(\frac{F_{21}^{H H}(X)-(-13)}{45.26667-(-13)}\right)^{p}+w_{4}^{p}\left(\frac{F_{22}^{H H}(X)-(-13.2)}{17.66667-(-13.2)}\right)^{p}\right]^{1 / p}
\end{aligned}
$$

Thus, problem (11) is obtained. In order to get numerical solutions, assume that $w_{1}^{p}=w_{2}^{p}=w_{3}^{p}=w_{4}^{p}=0.25$ and $\mathrm{p}=2$,

\begin{tabular}{|c|c|c|c|c|c|c|c|}
\hline & $d_{2}^{P I S^{H H}}$ & $d_{2}^{N I S^{H H}}$ & $F_{21}^{H H}(X)$ & $F_{22}^{H H}(X)$ & $x_{1}$ & $x_{2}$ & $x_{3}$ \\
\hline Min. $d_{2}^{P I S^{H H}}$ & $11.12^{*}$ & $0.35^{-}$ & 45.27 & -6.87 & 6.3 & $\begin{array}{l}3 . \\
2\end{array}$ & 0 \\
\hline $\operatorname{Max} . d_{2}^{N I S^{H F}}$ & 0.04 & $0.17^{*}$ & 33.92 & 11.77 & $\begin{array}{c}10 . \\
4\end{array}$ & 0. & 0 \\
\hline
\end{tabular}

Table 33. PIS payoff table of problem (11) when $\mathrm{p}=2$

$d_{2}^{*^{H H}}=(11.12249,0.1680895), d_{2}^{-H H}=(0.03948343031$, $0.3545651373)$.

Now, it is easy to compute step (10):

Maximize $\delta^{H H-D M_{L_{2}}}$

Subject to

$$
\begin{aligned}
& 3 x_{1}+5 x_{2}+x_{3} \leq 35,2 x_{1}-x_{2}+12 x_{3} \leq 20,5 x_{2}+6 x_{3} \leq \\
& 16, x_{1} \geq 1, x_{1}, x_{2}, x_{3} \geq 0 \\
& \left(\frac{d_{1}^{P I S}(X)-11.12249}{0.1680895-11.12249}\right) \geq \delta^{H H-D M_{L_{2}}} \\
& \left(\frac{0.03948343031-d_{1}^{N I S} S^{H H}}{0.03948343031-0.3545651373}\right) \geq \delta^{H H-D M_{L_{2}}}
\end{aligned}
$$

$\delta^{H H-D M_{L_{2}}} \in[0,1]$.

$\left(\frac{(10.38462+0.001)-x_{1}}{0.001}\right) \geq \delta^{H H-D M_{L_{2}}}$

$\left(\frac{x_{1}-(10.38462-0.001)}{0.001}\right) \geq \delta^{H H-D M_{L_{2}}}$

The maximum "satisfactory level" $\left(\delta^{H H-D M_{L_{2}}}=\right.$ zero $)$ is achieved for the solution $x_{1}^{*{ }^{H H}}=10.38462$, $x_{2}^{* H H}=0.7692308, x_{3}^{* H H}=$ zero.

\section{(Third Level):}

Obtain PIS and NIS payoff tables for problem $P^{H H}$ :

Table 34. PIS payoff table for problem $P^{H H}$

\begin{tabular}{lccccc}
\hline & $\boldsymbol{F}_{\mathbf{3 1}}^{\boldsymbol{H}}(\boldsymbol{X})$ & $\boldsymbol{F}_{\mathbf{3 2}}^{\boldsymbol{H}}(\boldsymbol{X})$ & $\boldsymbol{x}_{\mathbf{1}}$ & $\boldsymbol{x}_{\mathbf{2}}$ & $\boldsymbol{x}_{\mathbf{3}}$ \\
\hline $\operatorname{Max} . F_{31}^{H H}(X)$ & $87.54^{*}$ & 66.08 & 10.4 & 0.8 & 0 \\
$\operatorname{Max} . F_{32}^{H H}(X)$ & 87.54 & $66.08^{*}$ & 10.4 & 0.8 & 0 \\
\hline
\end{tabular}

PIS: $f^{*^{H H}}=(87.53846,66.07692)$

Table 35. NIS payoff table for problem $P^{H H}$

\begin{tabular}{lccccc}
\hline & $\boldsymbol{F}_{31}^{\boldsymbol{H}}(\boldsymbol{X})$ & $\boldsymbol{F}_{\mathbf{3 2}}^{\boldsymbol{H}}(\boldsymbol{X})$ & $\boldsymbol{x}_{\mathbf{1}}$ & $\boldsymbol{x}_{\mathbf{2}}$ & $\boldsymbol{x}_{\mathbf{3}}$ \\
\hline $\operatorname{Min} . F_{31}^{H H}(X)$ & $-0.4^{-}$ & 33.6 & 0 & 3.2 & 0 \\
$\operatorname{Min} F_{32}^{H H}(X)$ & 7.67 & $6.3^{-}$ & 0 & 0 & 1.67 \\
\hline
\end{tabular}

NIS: $f^{-L H}=(-0.4,6.3333)$

\begin{tabular}{|c|c|c|c|c|c|c|c|}
\hline & $d_{3}^{P I S^{H H}}$ & $d_{3}^{N I S^{H H}}$ & $F_{31}^{H H}(X)$ & $F_{32}^{H H}(X)$ & $x_{1}$ & $x_{2}$ & $x_{3}$ \\
\hline Min. $d_{3}^{P I S^{H H}}$ & $14.42^{*}$ & $0.4^{-}$ & 87.54 & 66.08 & $\begin{array}{c}10 . \\
4\end{array}$ & $\begin{array}{c}0.7 \\
7\end{array}$ & 0 \\
\hline $\operatorname{Max} . d_{3}^{N I S^{H I}}$ & 0.05 & $\begin{array}{l}0.9 E \\
-01^{*}\end{array}$ & 87.54 & 66.08 & $\begin{array}{c}10 . \\
4\end{array}$ & $\begin{array}{c}0.7 \\
7\end{array}$ & 0 \\
\hline
\end{tabular}

Next, construct equation and obtain the following equations:

$$
\begin{aligned}
& d_{p}^{P I S^{H H-D M_{L_{3}}}} \\
& =\left[w_{1}^{p}\left(\frac{113.2308-F_{11}^{H H}(X)}{113.2308-4}\right)^{p}+w_{2}^{p}\left(\frac{97.69231-F_{12}^{H H}(X)}{97.69231-(-3.2)}\right)^{p}+\right. \\
& w_{3}^{p}\left(\frac{45.26667-F_{21}^{H H}(X)}{45.26667-(-13)}\right)^{p}+w_{4}^{p}\left(\frac{17.66667-F_{22}^{H H}(X)}{17.66667-(-13.2)}\right)^{p}+ \\
& \left.w_{5}^{p}\left(\frac{87.53846-F_{31}^{H H}(X)}{87.53846-(-0.4)}\right)^{p}+w_{6}^{p}\left(\frac{66.07692-F_{32}^{H H}(X)}{66.07692-(6.3333)}\right)^{p}\right]^{1 / p} \\
& d_{p}^{N I S^{H H-D M}}{ }_{L_{3}} \\
& =\left[w_{1}^{p}\left(\frac{F_{11}^{H H}(X)-(4)}{113.2308-4}\right)^{p}+w_{2}^{p}\left(\frac{F_{12}^{H H}(X)-(1.8)}{97.69231-(1.8)}\right)^{p}+\right. \\
& w_{3}^{p}\left(\frac{F_{21}^{H H}(X)-(-13)}{45.26667-3}\right)^{p}+w_{4}^{p}\left(\frac{F_{22}^{H H}(X)-(-13.2)}{17.66667-(-13.2)}\right)^{p}+ \\
& \left.w_{5}^{p}\left(\frac{F_{31}^{H H}(X)-(-0.4)}{87.53846-(-0.4)}\right)^{p}+w_{6}^{p}\left(\frac{F_{32}^{H H}(X)-(6.3333)}{66.07692-(6.33333)}\right)^{p}\right]^{1 / p}
\end{aligned}
$$

Thus, problem (11) is obtained. In order to get numerical solutions, assume that $w_{1}^{p}=w_{2}^{p}=w_{3}^{p}=w_{4}^{p}=w_{5}^{p}=w_{6}^{p}=1 / 6$ and $\mathrm{p}=2$,

Table 36. PIS payoff table of problem (11) when $\mathrm{p}=2$

$d_{3}^{*^{H H}}=(14.42384,0.8510848 E-01)$,

$d_{3}^{-H H}=(0.04546254747,0.3838009497)$.

Now, it is easy to compute step (10):

Maximize $\delta^{\mathrm{HL}-D M_{L_{3}}}$

Subject to

$3 x_{1}+5 x_{2}+x_{3} \leq 35,2 x_{1}-x_{2}+12 x_{3} \leq 20,5 x_{2}+6 x_{3} \leq$

$\left(\frac{d_{3}^{P I S^{H H}}(X)-14.42384}{0.8510848 E-01-14.42384}\right) \geq \delta^{\mathrm{HL}-D M_{L_{3}}}$,

$\left(\frac{0.04546254747-d_{3}^{N I S^{H H}}(X)}{0.04546254747-0.3838009497}\right) \geq \delta^{\mathrm{HL}-D M_{L_{3}}}$

$\delta^{\mathrm{HL}-D M_{L_{3}}} \in[0,1]$.

$\left(\frac{(0+0.001)-x_{3}}{0.001}\right) \geq \delta^{\mathrm{HL}-D M_{L_{3}}},\left(\frac{x_{3}-(0-0.001)}{0.001}\right) \geq \delta^{\mathrm{HL}-D M_{L_{3}}}$

The maximum "satisfactory level"

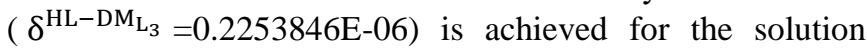
$\mathrm{x}_{1}^{*^{\mathrm{HH}}}=10.38461, \mathrm{x}_{2}^{*^{\mathrm{HH}}}=0.7692309, \mathrm{x}_{3}^{*^{\mathrm{HH}}}=0.9999998 \mathrm{E}-07$.

Note:

Numbers at Table (1) to Table (36) are approximated.

Table (37) presents a comparison among the proposed TOPSIS method, Global Criterion (GC) Method and the ideal objective vector (IOV). In general, the proposed TOPSIS algorithm is a good method to generate compromise solutions $($ at $\mathrm{p}=2)$. 
Table 37. Comparison among the proposed algorithm, the GC method and the vector of ideal solutions

\begin{tabular}{cccccc}
\hline Objective & $\begin{array}{c}\text { Proposed } \\
\text { TOPSIS } \\
\text { Algorithm } \\
\text { method } \\
(\mathbf{p}=\mathbf{2})\end{array}$ & $\begin{array}{c}\text { Global } \\
\text { Criterion } \\
\text { (GC) } \\
\text { Method }\end{array}$ & \multicolumn{2}{c}{$\begin{array}{c}\text { Ideal Objective } \\
\text { Vector }\end{array}$} \\
\cline { 3 - 6 } & & & & & \\
$\mathrm{P}$ & & & $\mathrm{PIS}$ & $\mathrm{NIS}$ \\
& $\mathrm{F}_{1}^{\mathrm{LL}}$ & 23.230778 & 5.6678893 & 24 & -2.4 \\
& $\mathrm{~F}_{2}^{\mathrm{LL}}$ & 59.465616 & 1.0033662 & 59.4615 & 4.3 \\
$\mathrm{P}$ & $\mathrm{F}_{1}^{\mathrm{HL}}$ & 55.384638 & 13 & 55.38462 & -1.4 \\
& $\mathrm{~F}_{2}^{\mathrm{HL}}$ & 62.0090232 & 15 & 62 & 5.333 \\
$\mathrm{P}^{\mathrm{LH}}$ & $\mathrm{F}_{1}^{\mathrm{LH}}$ & 13 & 3 & 13 & -3.4 \\
& $\mathrm{~F}_{2}^{\mathrm{LH}}$ & 55 & 11 & 58.4615 & 3.3333 \\
$\mathrm{P}^{\mathrm{HH}}$ & $\mathrm{F}_{1}^{\mathrm{HH}}$ & 87.538418 & 13 & 87.5384 & -0.4 \\
& $\mathrm{~F}_{2}^{\mathrm{HH}}$ & 66.076897 & 20 & 66.0769 & 6.3333 \\
\hline & & & & & \\
\hline
\end{tabular}

\section{CONCLUSIONS}

This paper extended TOPSIS approach to find compromise solutions for the multi-level multi-objective decision making problems with rough parameters in the objective functions (RMLMODM). A new hybrid algorithm based on modified TOPSIS method and the "Lower \& Upper" approximations method for solving RMLMODM problems is proposed. Also, an illustrative numerical example is solved and compared the compromise solutions of the proposed algorithm with the vector of ideal solutions and the traditional global criterion method. The engineers and the scientists can apply the introduced hybrid algorithm to various practical RMLMODM problems to obtain numerical solutions

\section{REFERENCES}

[1] Pawlak Z. (1982). Rough sets. basic notions. International Journal of Computer and Information Sciences 11: 341-356. https://doi.org/10.1007/BF01001956

[2] Hamzehee A, Yaghoobi MA, Mashinchi M. (2014). Linear programming with rough interval coefficients. Intelligent and Fuzzy Systems 26: 1179-1189. https://doi.org/ 10.3233/IFS-130804

[3] Abou-El-Enien THM. (2013). TOPSIS algorithms for multiple objectives decision making: Large scale programming approach. LAP LAMBERT Academic Publishing.

[4] El-Feky SF, Abou-El-Enien THM. (2018). Compromise solutions for rough multiple objective decision making problems. Australian Journal of Basic and Applied Sciences $\quad 12(7)$ : 112-119. https://doi.org/10.22587/ajbas.2018.12.7.18

[5] Lai YJ, Liu TY, Hwang CL. (1994). TOPSIS for MODM. European Journal of Operational Research 76: 486-500. https://doi.org/10.1016/0377-2217(94)90282-8

[6] Abo-Sinna MA, Abou-El-Enien THM. (2005). An algorithm for solving large scale multiple objective decision making problems using TOPSIS approach. AMSE Journals, Advances in Modelling and Analysis A 42(6): 31-48.

[7] Abou-El-Enien THM, El-Feky SF. (2015). BI-level, multi-level multiple criteria decision making and TOPSIS approach-Theory, Applications and Software: A literature review (2005-2015). Global Journal of Advanced Research 2(7): 1179-1195.

[8] Hwang CL, Masud ASM. (1979). Multiple objective decision making methods and applications. SpringerVerlag.

[9] Liu GP Yang JB, Whidborne JF. (2003). Multiobjective Optimisation and Control. Research Studies Press LTD.

[10] Zeleny M. (1982). Multiple Criteria Decision Making. McGraw-Hill.

[11] Bellman RE, Zadeh LA. (1970). Decision-making in fuzzy environment. Management Science, B 17: 141-164.

[12] Dauer P, Osman MSA. (1985). Decomposition of the parametric space in multiobjective convex programs using the generalized Tchebycheff norm. Journal of Mathematical Analysis and Applications 107(1): 156166.

[13] Dubois JD, Prade A. (1980). Fuzzy sets and systems: Theory and applications. Academic Press.

[14] Pramanik ST, Roy K. (2006). Fuzzy goal programming approach to multilevel programming problems. European Journal of Operational Research 176: 11511166. https://doi.org/10.1016/j.ejor.2005.08.024

[15] Sinha S. (2003). Fuzzy programming approach to multilevel programming problems. Fuzzy Sets and Syst 136: 189-202. https://doi.org/10.1016/S0165-0114(02)003627 